\title{
Electromagnetic scattering from very rough random surfaces and deep reflection gratings
}

\author{
J. M. Soto-Crespo and M. Nieto-Vesperinas \\ Instituto de Optica, Consejo Superior de Investigaciones Cientificas, Serrano, 121, 28006-Madrid, Spain
}

Received July 4, 1988; accepted October 14, 1988

\begin{abstract}
A theoretical study of electromagnetic wave scattering from deep perfectly conducting one-dimensional random rough surfaces and reflection gratings is performed by means of the extinction theorem. The scattering equations are solved numerically (instead of being solved by the usual analytical procedures, which are valid only for slight corrugations). This permits us to obtain an exhaustive collection of results for the mean scattered intensity as a function of polarization and surface parameters. In particular, Lambertian scattering and enhanced backscattering are predicted for random surfaces. Also, the range of validity of the Kirchhoff approximation is established for random surfaces whose correlation length is comparable with or smaller than the wavelength. Concerning gratings, generalizations of the blaze for large angles of incidence, large periods, and arbitrary shapes are obtained. Finally, it is shown that the blaze of the antispecular order for gratings is at the root of the enhanced backscattering for random surfaces.
\end{abstract}

\section{INTRODUCTION}

The study of the scattering of light and other electromagnetic waves from rough surfaces has been an active task for many years. Despite the large amount of research reported on this subject there are still several aspects that are not understood well yet. For instance, the range of validity of the Kirchhoff approximation ${ }^{1-4}(\mathrm{KA})$ is not well known yet for random surfaces. It is commonly believed that this validity requires that $T$, the correlation length of the surface height, be such that $T \gg \lambda$ ( $\lambda$ is the wavelength) and $\sigma$, the root-mean-square height, be such that $\sigma / T$ is small. Also, scattering models that account rigorously for the interaction process apply to slightly rough surfaces, as they are perturbative, in forms derived either from the known Rayleigh hypothesis ${ }^{5-8}$ or from the extinction theorem ${ }^{9-16}(E T)$, which constitutes an exact boundary condition. (A review may be found in Refs. 17 and 18.) Therefore details of the behavior of the mean scattered intensity as a function of polarization, $T, \sigma$, and $\lambda$ are not well known yet. This incomplete knowledge is due both to the difficulty of making predictions with an accurate theory capable of covering large $\sigma$ and small $T$ and to the lack of sufficient experimental data.

Recently, some new experiments ${ }^{19,20}$ yielded additional results, including the effect of enhanced backscattering from highly conductive deep random surfaces for both $s$ and $p$ polarizations. This effect appears to be related to the phenomenon of weak localization of photons in random media ${ }^{21-25}$ and also has been predicted for shallow finitely conductive surfaces under $p$ polarization. ${ }^{26}$ (We adopt the convention of considering $s$ or $p$ polarization according to whether the electric vector is perpendicular or parallel to the plane of incidence.)

No theoretical study (to our knowledge) has been able to account for moderately and extremely rough surfaces; thus enhanced backscattering from deep surfaces has not been accounted for. However, some preliminary results on this phenomenon were obtained recently. 27,28

As for gratings, the situation is much more satisfactory from both the theoretical and the experimental points of view. ${ }^{29,30}$ However, some new results have also been obtained and are presented in this paper, as indicated below, specifically in connection with enhanced backscattering.

In the present paper we attempt to fill some gaps between experimental results and some of the available theory. We examine in greater detail the preliminary results of Ref. 27, in which the ET was used as a boundary condition for finding the sources.

The corresponding equations are solved numerically instead of analytically (which is possible only in the KA or in a few orders of perturbation). Perfectly conductive random surfaces are generated by means of a Monte Carlo procedure. The surfaces are one dimensional; thus no depolarization effects can be accounted for. However, we believe that for given values of $\sigma$ and $T$, all the essentials (other than depolarization) can be interpreted adequately, in particular, the phenomenon of enhanced backscattering, the behavior of the mean intensity in the backscattering direction as $\lambda$ varies, and the Lambertian regime at a certain combination of $T$ and $\sigma$.

As for gratings, new results on blaze ${ }^{31-36}$ have been obtained. ${ }^{37}$ We have confirmed that the enhancement of the antispecular order can be observed for any shape and any number of propagating orders ${ }^{34,36}$ and regardless of how large the period or the angle of incidence is (provided, of course, it is not close to the grazing incidence). Gratings can be designed easily with enhancement in all antispecular orders.

Also, we show that for any given profile both the specular and the antispecular orders have the highest probability of being enhanced and that, if the unit cell has no symmetry, then the antispecular order has an even higher probability of blaze than does the specular order. By considering the averages of diffracted intensities from several gratings, we show the intimate connection between the enhancement of the antispecular order and the enhanced backscattering from random surfaces.

It should be remarked that the results presented here still 
have two limitations for random surfaces, because of the limited number of sampling points of the surface with which it is possible to work. First, the theory is restricted to onedimensional surfaces; second, the illuminated length of the surface is rather small, and hence the resolution of the scattered intensity is not high.

\section{RANDOM ROUGH SURFACES}

\section{A. Scattering Equations}

Let us consider a linearly polarized electromagnetic wave incident at an angle $\theta_{0}$ upon a surface $z=D(x)$ separating a vacuum from a perfect conductor (see Fig. 1 and Appendix A). Since the surface variation takes place only in the $x$ coordinate, we have to consider the problem only in the plane of incidence. With reference to Fig. 1, the incident electric vector is, for $s$ polarization,

$$
\mathbf{E}^{(i)}(\mathbf{r})=\hat{j} E^{(i)} \exp \left[i\left(K_{0} x-q_{0} z\right)\right],
$$

where $\mathbf{r}=(x, z), \hat{j}$ is the unit vector along $0 Y, E^{(i)}$ is a complex constant amplitude, and

$$
\begin{gathered}
K_{0}=k \sin \left(\theta_{0}\right), \\
q_{0}=k \cos \left(\theta_{0}\right)
\end{gathered}
$$

are the components of the incident wave vector; $k=2 \pi / \lambda$. A time dependence $\exp (-i \omega t)$ is suppressed everywhere.

The scattered field at any point above the surface is given by Eq. (A16) (see Appendix A) and is linearly polarized along the $\hat{j}$ direction. Therefore the one-dimensional surface $z=D(x)$ does not depolarize the $s$ wave. The electric current density component $J_{Y}\left[x^{\prime}, D\left(x^{\prime}\right)\right]$ representing the sources in Eq. (A16) is obtained from the boundary condition, Eq. (A15), evaluated at $\mathbf{r}=[x, D(x)]$, given by the ET.

Since we are interested in the far-zone intensity, we take the asymptotic expression for the Hankel function $H_{0}\left(k \mid \mathbf{r}_{>}\right.$ $-\mathbf{r}^{\prime} \mid$ ) (cf. Ref. 38) and expand $\left|\mathbf{r}_{>}-\mathbf{r}^{\prime}\right| \cong r_{>}-\left[x^{\prime} \sin (\theta)+z^{\prime}\right.$ $\cos (\theta)$ ], obtaining

$$
\begin{aligned}
& E^{(s)}(\theta)=\left(\frac{-\pi k \sqrt{2}}{c \sqrt{\pi k r_{>}}}\right) \exp \left[i\left(k r_{>}-\pi / 2\right)\right] \int_{-\infty}^{\infty} J_{y}\left[x^{\prime}, D\left(x^{\prime}\right)\right] \\
& \times \exp \left\{-i k\left[x^{\prime} \sin \theta+D\left(x^{\prime}\right) \cos \theta\right]\right\}\left[1+\left(\frac{\mathrm{d} D}{\mathrm{~d} x^{\prime}}\right)^{2}\right]^{1 / 2} \mathrm{~d} x^{\prime},
\end{aligned}
$$

where

$$
\begin{aligned}
& x=r_{>} \sin (\theta), \\
& z=r_{>} \cos (\theta),
\end{aligned}
$$

$\theta$ being the observation angle. In practice, a finite length $L$ is illuminated; therefore the $x$ interval in Eq. (A15), (A16), or (3) is extended to the $L$ integral only.

Let $I_{0}$ represent the total power flow or integrated intensity of the incident wave:

$$
I_{0} \propto\left|E^{(i)}\right|^{2} L \cos \left(\theta_{0}\right) ;
$$

then the mean scattered intensity, normalized to the incident power flow, will be

$$
\frac{1}{I_{0}}\left\langle I_{s}^{(s)}(\theta)\right\rangle=\frac{1}{I_{0}} r_{>}\left\langle\left|E^{(s)}(\theta)\right|^{2}\right\rangle .
$$

By introducing Eq. (3) and relation (5) into Eq. (6), we obtain the normalized mean scattered intensity in the far zone for $s$ waves:

$$
\begin{aligned}
& \frac{1}{I_{0}}\left\langle I_{s}^{(s)}(\theta)\right\rangle=\frac{2 \pi k}{c^{2}} \frac{1}{\left|E^{(i)}\right|^{2} L \cos \theta_{0}} \\
& \quad \times\left\langle\left|\int_{-\infty}^{\infty} \mathrm{d} x^{\prime} J_{y}\left[x^{\prime}, D\left(x^{\prime}\right)\right] \exp \left\{-i k\left[x^{\prime} \sin \theta+D\left(x^{\prime}\right) \cos \theta\right]\right\}\right|^{2}\right\rangle .
\end{aligned}
$$

Equation (7) is the expression that we shall use in our calculations for $s$ waves.

For $p$ polarization, the incident wave is represented by the magnetic vector,

$$
\mathbf{H}^{(i)}(r)=\hat{j} H^{(i)} \exp \left[i\left(K_{0} x-q_{0} z\right)\right],
$$

$H^{(i)}$ being a complex constant amplitude.

The scattered field above the surface is given by Eq. (A21) of Appendix A and, once again, does not suffer depolarization. The electric current density component $J_{x}\left[x^{\prime}, D\left(x^{\prime}\right)\right]$ representing the sources in Eq. (A21) is obtained from the boundary condition, Eq. (A19), evaluated at $\mathbf{r}=[x, D(x)]$.

The scattered field in the far zone is expressed in a way analogous to that for $s$ polarization:

$$
\begin{aligned}
H^{(s)}(\theta)= & \frac{\pi i k}{c}\left(2 / \pi k r_{>}\right)^{1 / 2} \exp (-i 3 \pi / 4) \exp \left(i k r_{>}\right) \\
& \times \int_{-\infty}^{+\infty} \mathrm{d} x^{\prime} J_{x}\left[x^{\prime}, D\left(x^{\prime}\right)\right] \\
& \times \exp \left\{-i k\left[x^{\prime} \sin \theta+D\left(x^{\prime}\right) \cos \theta\right]\right\} \\
& \times\left(\cos \theta-\frac{\mathrm{d} D}{\mathrm{~d} x^{\prime}} \sin \theta\right)\left[1+\left(\frac{\mathrm{d} D}{\mathrm{~d} x^{\prime}}\right)^{2}\right]^{1 / 2}
\end{aligned}
$$

The normalized mean scattered intensity in the far zone for $p$ waves is expressed as

$$
\begin{aligned}
\frac{1}{I_{0}}\left\langle I_{p}{ }^{(s)}(\theta)\right\rangle= & \frac{2 \pi k}{c^{2}} \frac{1}{\left|H^{(i)}\right|^{2} L \cos \theta_{0}}\langle| \int_{-\infty}^{+\infty} \mathrm{d} x^{\prime} J_{x}\left[x^{\prime}, D\left(x^{\prime}\right)\right] \\
& \times \exp \left\{-i k\left[x^{\prime} \sin \theta+D\left(x^{\prime}\right) \cos \theta\right]\right\} \\
& \left.\times\left.\left(\cos \theta-\frac{\mathrm{d} D}{\mathrm{~d} x^{\prime}} \sin \theta\right)\left[1+\left(\frac{\mathrm{d} D}{\mathrm{~d} x^{\prime}}\right)^{2}\right]^{1 / 2}\right|^{2}\right\rangle .
\end{aligned}
$$

Equation (10) is used in our calculation for $p$ waves.

Equations (7) and (10) both must satisfy the unitarity condition:

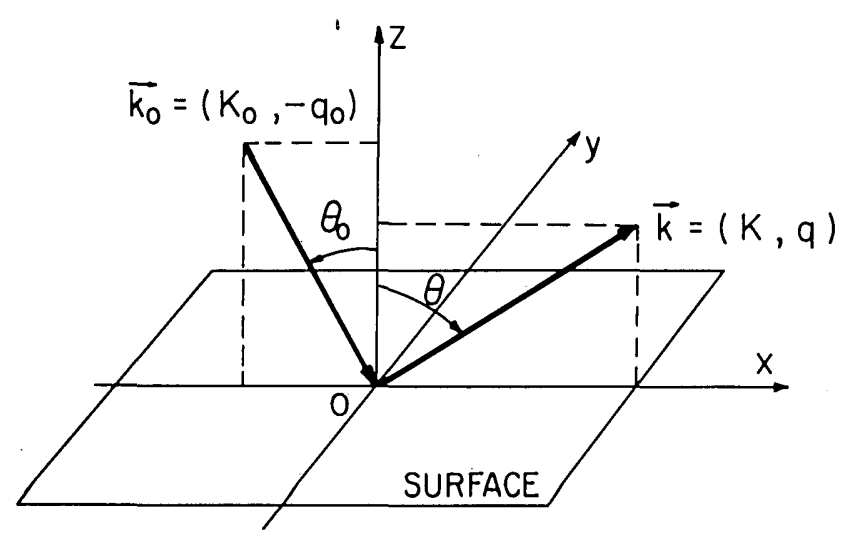

Fig. 1. Illustration of the scattering geometry. 


$$
\frac{1}{I_{0}} \int_{-\pi / 2}^{\pi / 2}\left\langle I_{\{p\}}{ }^{(s)}(\theta)\right\rangle \mathrm{d} \theta=1 .
$$

The above equations contain all the information that is needed to solve the scattering problem for random surfaces. In Subsection 1.B a certain model for the profile $z=D(x)$ is made, and then the scattering equations are solved. This is done numerically, since an exact analytical method seems impossible.

\section{B. Numerical Results}

The surface profile $z=D(x)$ is assumed to be a statistically homogeneous random process with zero mean and a normal probability-density function. The covariance function $C(\tau)$ is assumed to be Gaussian:

$$
C(\tau)=\langle D(x) D(x+\tau)\rangle=\sigma^{2} \exp \left(-\tau^{2} / T^{2}\right),
$$

$T$ being the correlation length and $\sigma^{2}$ being the variance or mean-square deviation of the surface from the line $z=0$. With these statistics, all odd moments vanish and the higher order ones can be expressed as combinations of $C(\tau)$.

The surface profiles are generated by following the Monte Carlo procedure used in Ref. 39; that is, a sequence of random numbers (typically $10^{5}$ ) with normal statistics, zero mean, and unity variance is constructed from another series of random numbers distributed uniformly in $(0,1)$ generated directly by the computer. The former sequence is then scaled to the desired variance $\sigma^{2}$, and the resulting sequence, say, $\left\{y_{k}\right\}$, is correlated with a Gaussian, $(2 / \sqrt{\pi} T) \exp \left[-2(k / T)^{2}\right]$, to obtain the appropriate surface profile sequence $\left\{z_{k}=\right.$ $\left.D\left(x_{k}\right)\right\}$ with a Gaussian correlation function.

For a given incident plane wave with either $s$ or $p$ polarization, the corresponding induced current density is obtained by solving the linear system represented by either Eq. (A15) or Eq. (A19). The normalized mean scattered intensity in the far zone is then obtained from either Eq. (7) or Eq. (10) by averaging over many samples of length $L$, which represents the effective interval of $x$ integration. In our case, we averaged over 200 samples; with each sample of length $L$ being obtained by extraction of different segments of the sequence $\left\{z_{k}\right\}$ with typically $N=221$ sampling points, so that $L=(N-1) \delta, \delta$ being the sampling interval. The electric current density, for each sample and at each incidence angle, then was evaluated at each of the 220 sampling points, which are at the midpoint of each of the resulting 220 intervals. The mean scattered intensities obtained by this procedure still contained ripples; a further smoothing was performed by using a weighted average of every three consecutive sampling points. The finite averaging record also produced asymmetric curves for $\theta_{0}=0^{\circ}$, which were symmetrized subsequently by averaging every two values of the resulting mean intensity at scattering angles $\theta$ and $-\theta$. Calculations were done on a CDC-Cyber 180/855 computer. The unitarity condition [Eq. (11)], both its average and its variance from the 200 samples, was used as a criterion of numerical consistency of the results.

An additional smoothing of the resulting intensities may be obtained by introducing a Gaussian window in the incident field, namely, by using an incident Gaussian beam with almost zero amplitude at both extremes of the $L$ interval. However, the influence on the ripples of the mean scattered intensities associated with the Monte Carlo procedure is

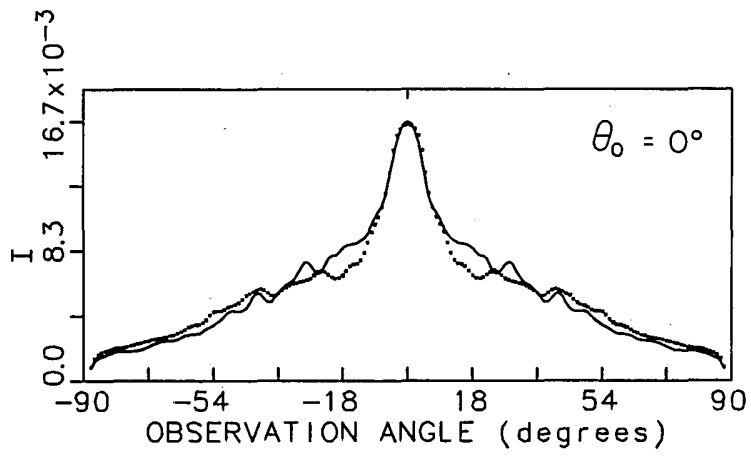

(a) $\begin{aligned} \sigma & =1.50 \lambda \\ T & =1.80 \lambda\end{aligned}$

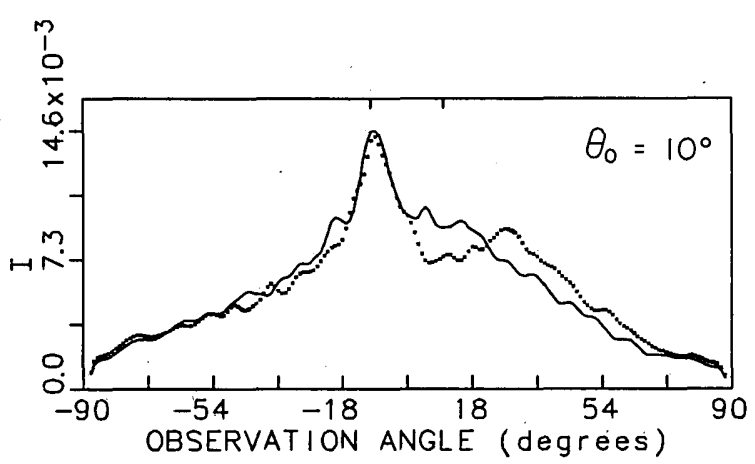

(b)

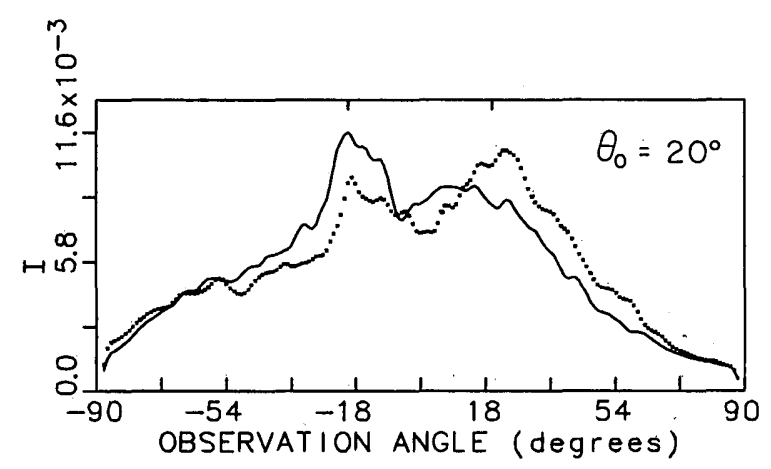

(c)

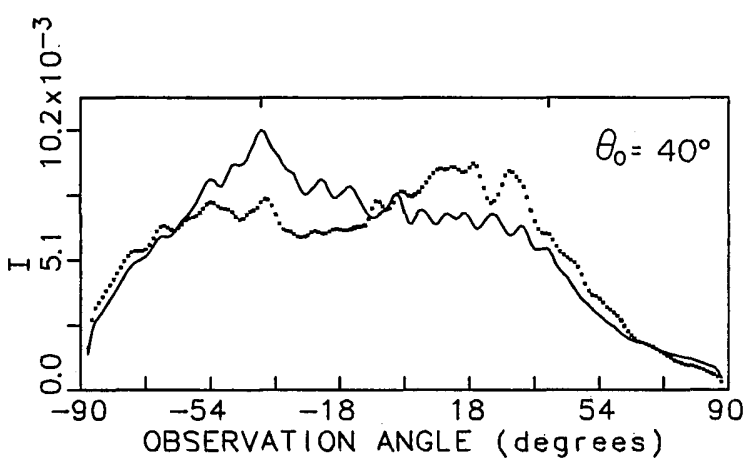

(d)

Fig. 2. Mean scattered intensity from a random surface with $T=$ $1.8 \lambda$ and $\sigma=1.5 \lambda$ for $s$ polarization (solid curves) and for $p$ polarization (dotted curves): (a) $\theta_{0}=0^{\circ}$, (b) $\theta_{0}=10^{\circ}$, (c) $\theta_{0}=20^{\circ}$, (d) $\theta_{0}=$ $40^{\circ}$. The two upper peaks mark the backscattering (left) and specular (right) directions. Unitarity of these results is kept within $4 \%$ error. 


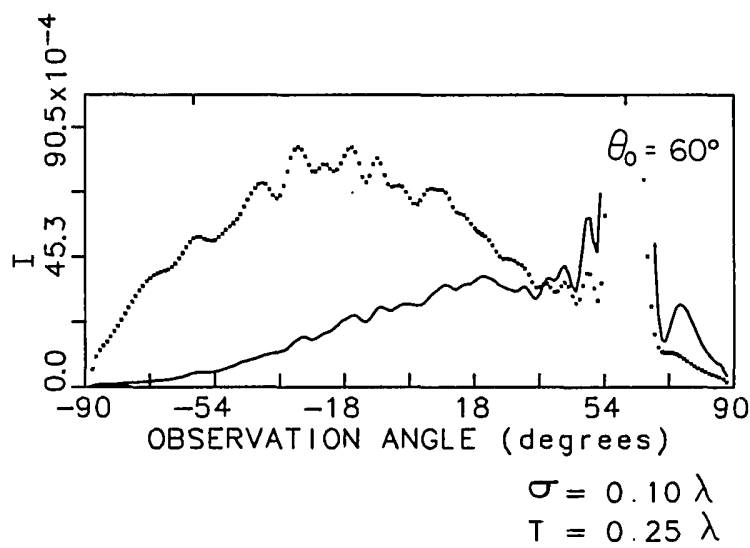

Fig. 3. Same as Fig. 2 for $\sigma=0.1 \lambda, T=0.25 \lambda$, and $\theta_{0}=60^{\circ}$.

more important than that due to edge diffraction. Moreover, in cases in which the sidelobes of the specular peak are quite strong and can mask the diffuse part of the scattered intensity, it is convenient to subtract the coherent part (either $\left|\left\langle E^{(s)}(\theta)\right\rangle\right|^{2}$ or $\left|\left\langle H^{(s)}(\theta)\right\rangle\right|^{2}$, depending on whether it is $s$ or $p$ polarization), from the total mean scattered intensity.

For $T \simeq \lambda$ we take $L=22 \lambda$ (but $L$ can be made three times larger for $\sigma / T \ll 1$ ). For $T=0.2 \lambda$ and $T=0.25 \lambda$ (see Figs. 3 and 4 below), we use $L=12 \lambda$. For $T>3 \lambda, L=60 \lambda$. Therefore the corresponding lengths of the sampling intervals are $0.1 \lambda, 0.05 \lambda$, and $0.27 \lambda$, respectively, for these three cases. With these values we get 10 or more asperities of the surface at each sample.

Even though the calculations presented here are one dimensional, they have a strong qualitative similarity with the experimental mean scattered intensities obtained from twodimensional rough surfaces in Ref. 20. Figures 2(a), 2(b), 2 (c), and 2(d) show our calculated mean scattered intensity, corresponding to $\theta_{0}$ values of $0^{\circ}, 10^{\circ}, 20^{\circ}$, and $40^{\circ}$, respectively, for a one-dimensional surface with $T=1.8 \lambda$ and $\sigma=$ $1.5 \lambda$ (solid curves, $s$ waves; dotted curves, $p$ waves). Figures 2(a), 2(c), and 2(d) for $s$ waves are similar to Figs. 9, 10, and 11 , respectively, of Ref. 20 for $T=2.2 \lambda$ and $\sigma=1.6 \lambda$, indicating the existence of enhanced backscattering. (The convention of angles in Ref. 20 is such that those figures appear reflected with respect to ours.) Our results, however, lack the resolution obtained in experiments, as we are constrained to using a finite number of sampling points. A quantitative comparison cannot strictly be made, as our theoretical model is one dimensional. In addition, the same normalization for both kinds of data should be required.

In the range of parameters that we have worked with there are many cases in which the results for $s$ waves are different from those for $p$ waves, although we would expect that, as $\sigma$ increases, both results would tend to be similar (cf. Ref. 40). There is a regime in which this difference is markedly interesting, namely, when $\sigma$ is small enough to produce a strong difference between $s$ and $p$ scattered waves, but in which $T$ is also small (and comparable to $\sigma$ ), so that the KA is not valid and resonant scattering takes place. Figure 3 shows the $s$ and $p$ calculated mean scattered intensities for $T=0.25 \lambda$ and $\sigma=0.1 \lambda$ at $\theta_{0}=60^{\circ}$. The strong specular peak appearing in both polarizations has been cut. It is remarkable that the diffuse halo of $p$ waves is larger than that of $s$ waves. This, once again, is in good qualitative agreement with recent experimental results. ${ }^{41}$ As $\sigma$ increases, at similar low $T$, the difference between $s$ and $p$ waves becomes larger. Figures $4(\mathrm{a})$ and 4 (b) show the mean scattered intensities obtained for $T=0.2 \lambda$ and $\sigma=0.2 \lambda$ at $\theta_{0}=10^{\circ}$ and $50^{\circ}$, respectively. Now the $p$ wave does not yield an appreciable specular peak, in contrast to the $s$ wave, which still does (note the broad width of this peak, which is due to the finite extent $L$ of the samples). Also, as $\theta_{0}$ increases, the tiny specular peak still observed at $\theta_{0}=10^{\circ}$ for $p$ waves tends to disappear (it is not seen at $\theta_{0}=50^{\circ}$, and, although the results are not shown here, it is not seen at $30^{\circ}$ either). Moreover, the scattering halo of the $p$ wave appears increasingly skewed toward the backscattering direction as $\theta_{0}$ increases. Of course, at grazing incidence, everything is specularly reflected.

The enhanced backscattering peak appears more distinguishable with respect to the halo for $\theta_{0} \leq 20^{\circ}$ and decreases as $\theta_{0}$ increases. This can be seen again in Figs. 2(a)-2(d) as well as in Figs. 5(a), 5(b), and 5(c), which correspond to $T=$ $2.5 \lambda$ and $\sigma=1.7 \lambda$ for $\theta_{0}=0^{\circ}, 10^{\circ}, 20^{\circ}$, respectively. Of course, the central peak shown in Fig. $5(\mathrm{a})$ at $\theta_{0}=0^{\circ}$, for both $s$ and $p$ polarizations, is backscattering. Note that for $\sigma$ as large compared with $T$ as in Figs. 5(a)-5(c), there is no appreciable difference between $s$ and $p$ waves. This seems to confirm that, for large $\sigma, s$ and $p$ scattering must be similar (in connection with the fact that no polaritions are excited by $p$ waves at large corrugations). From this result it is also concluded that the backscattering peak increases as $\sigma / T$ increases.

Within the range of values of $T$ that we are working with $(0.2 \leq T / \lambda \leq 5)$ we observe that, when $\sigma / T$ is kept constant, at $\theta_{0} \leq 20^{\circ}$ the value of the backscattering peak slightly

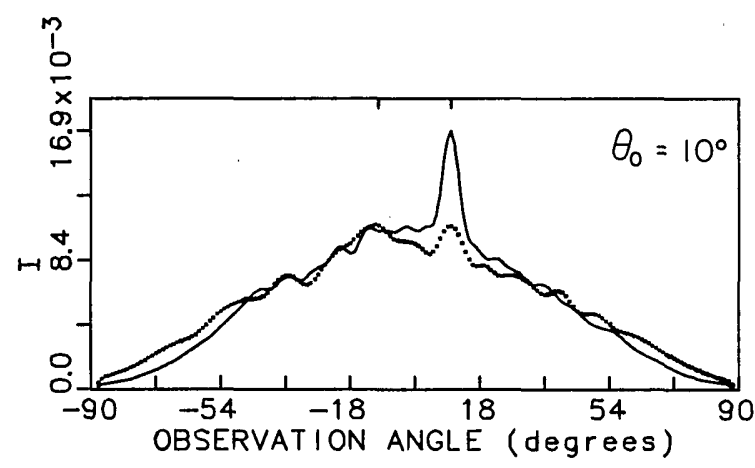

(a)
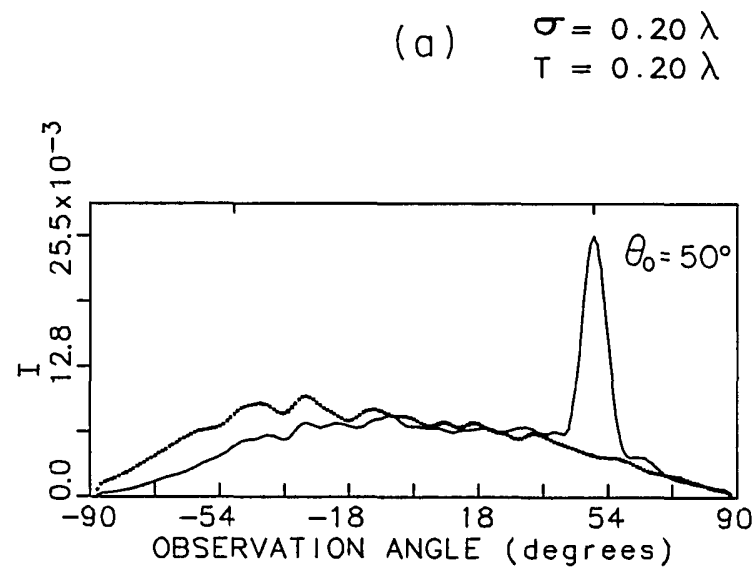

(b)

Fig. 4. Same as Fig. 2 for $T=0.2 \lambda$ and $\sigma=0.2 \lambda$. (a) $\theta_{0}=10^{\circ}$, (b) $\theta_{0}$ $=50^{\circ}$. 


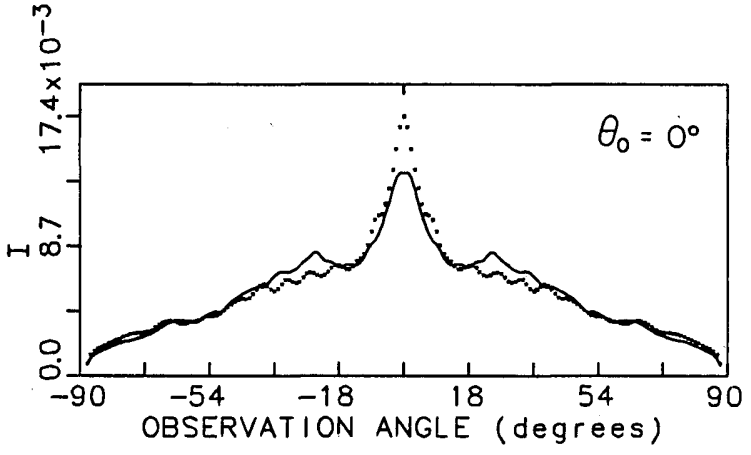

(a) $\begin{aligned} \sigma & =1.70 \lambda \\ T & =2.50 \hat{\lambda}\end{aligned}$

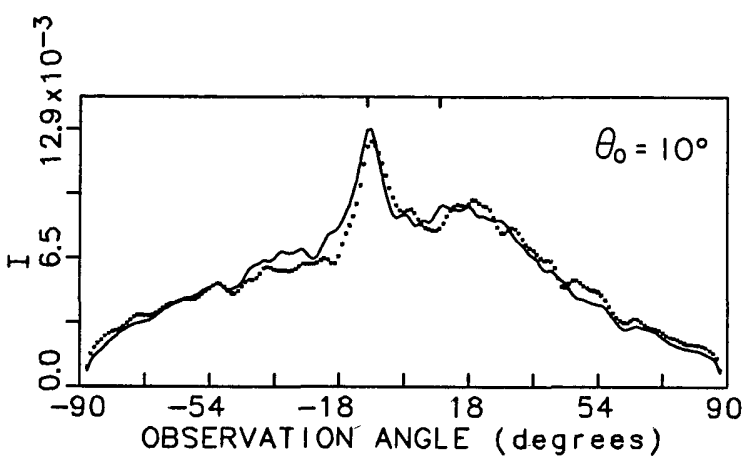

(b)

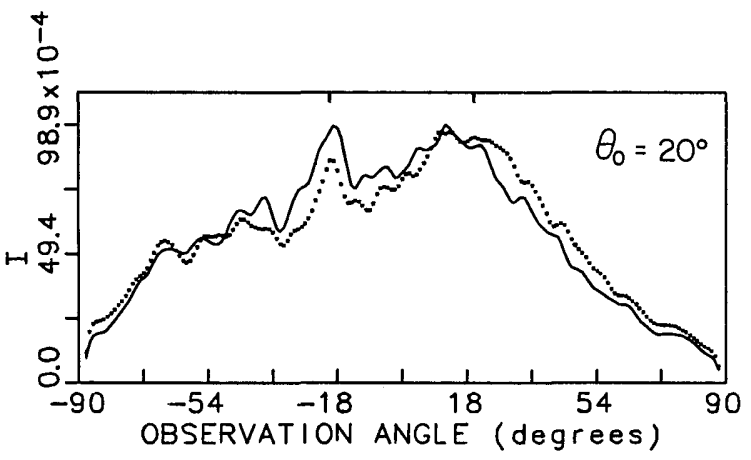

(c)

Fig. 5. Same as Fig. 2 for $T=2.5 \lambda$ and $\sigma=1.7 \lambda$. (a) $\theta_{0}=0^{\circ}$, (b) $\theta_{0}$ $=10^{\circ}$, (c) $\theta_{0}=20^{\circ}$.

increases as $\lambda$ decreases (i.e., as both $\sigma / \lambda$ and $T / \lambda$ increase). This is confirmed through comparison of Figs. 2(a) and 2(b) (for $\sigma=1.5 \lambda$ and $T=1.8 \lambda$ ) with Figs. 6(a) and 6(b) (for $\sigma=$ $0.5 \lambda$ and $T=0.5 \lambda$ ) and with Figs. 7(a) and 7(b) (for $\sigma=\lambda$ and $T=\lambda$ ). [Note that $\sigma / T$ is slightly lower in Fig. 2(a) than in Figs. 6(a) and 7(a).] However, its enhancement with respect to the background halo increases markedly as $\lambda$ decreases, at constant $\sigma / T$. Also, for constant $\sigma / T$, the value of the backscattering peak at incidence angles $\theta_{0}>20^{\circ}$ decreases as $\lambda$ decreases. This can be seen by comparing Figs. 2(c) and 2(d) with Figs. $6(\mathrm{c})$ and $6(\mathrm{~d})(\sigma=0.5 \lambda, T=0.5 \lambda)$ and with Figs. 7(c) and 7(d) $(\sigma=\lambda, T=\lambda)$. Even the results for the case in which $\sigma=0.2 \lambda$ and $T=0.2 \lambda$ fit in the above remarks [see Figs. 4(a) and 4(b); see also Fig. 1(a) of Ref. 27]. The increase in the value of the backscattering peak as $\lambda$ decreases while $\sigma / T$ is kept constant in the regime of parame-
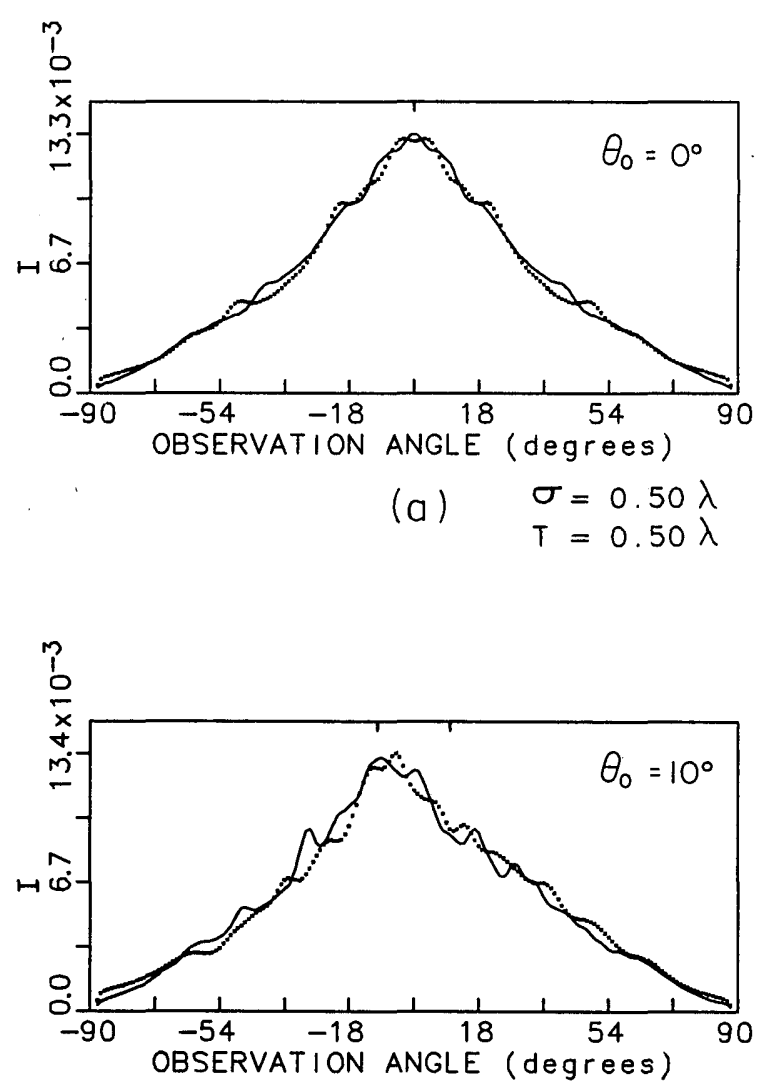

(b)

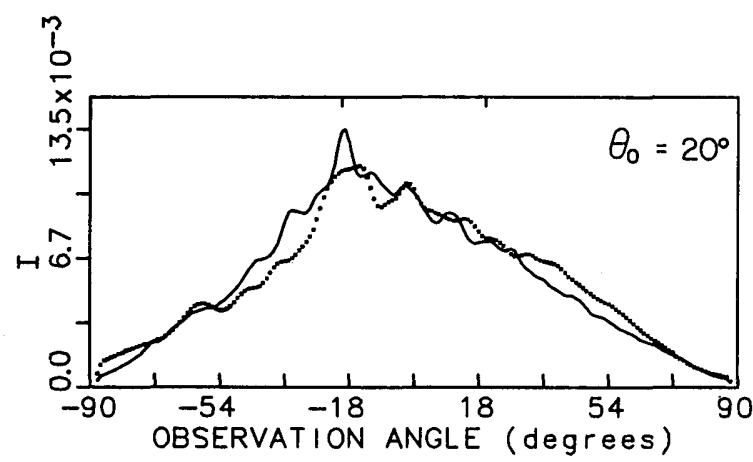

(c)

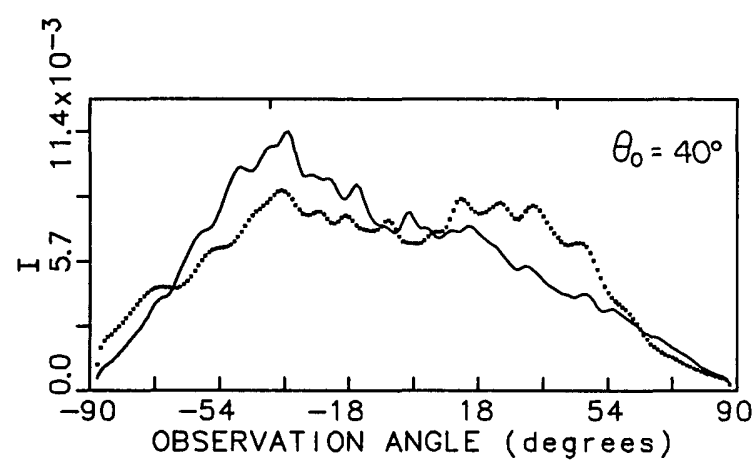

(d)

Fig. 6. Same as Fig. 2 for $T=0.5 \lambda$ and $\sigma=0.5 \lambda$. (a) $\theta_{0}=0^{\circ}$, (b) $\theta_{0}$ $=10^{\circ}$, (c) $\theta_{0}=20^{\circ}$, (d) $\theta_{0}=40^{\circ}$. 


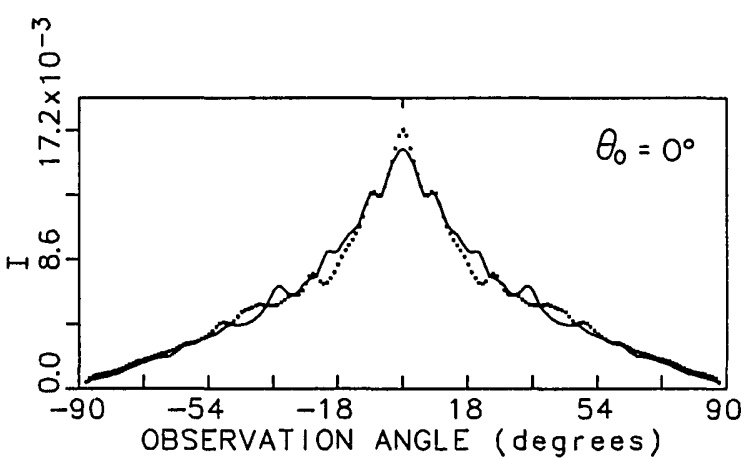

(a) $\begin{aligned} & \sigma=1.00 \lambda \\ & T=1.00 \lambda\end{aligned}$

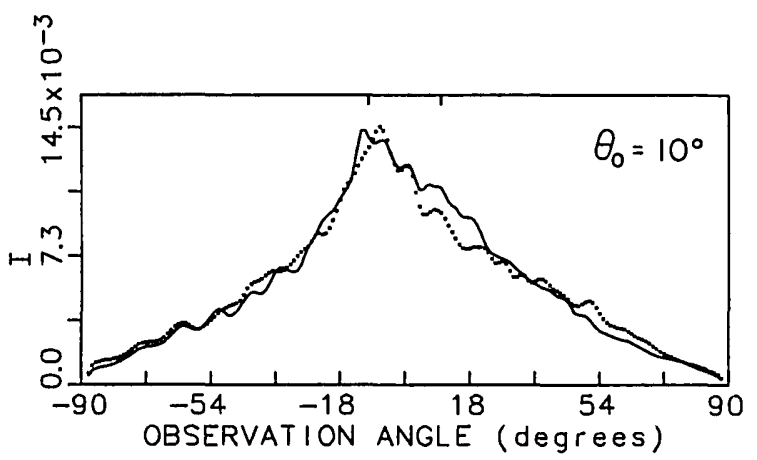

(b)

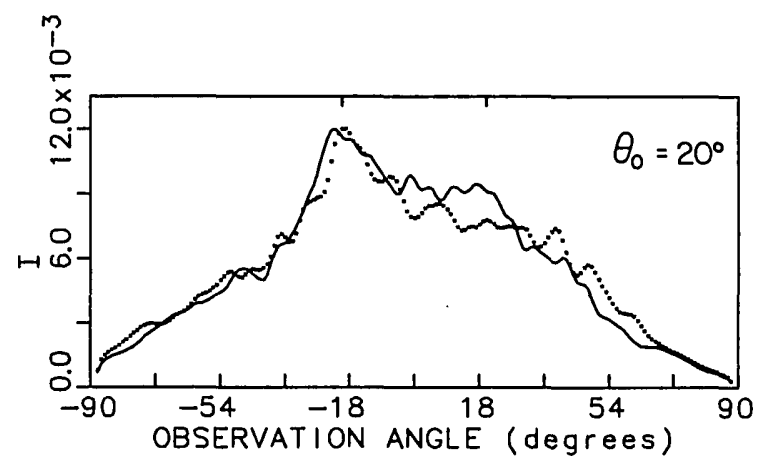

(c)

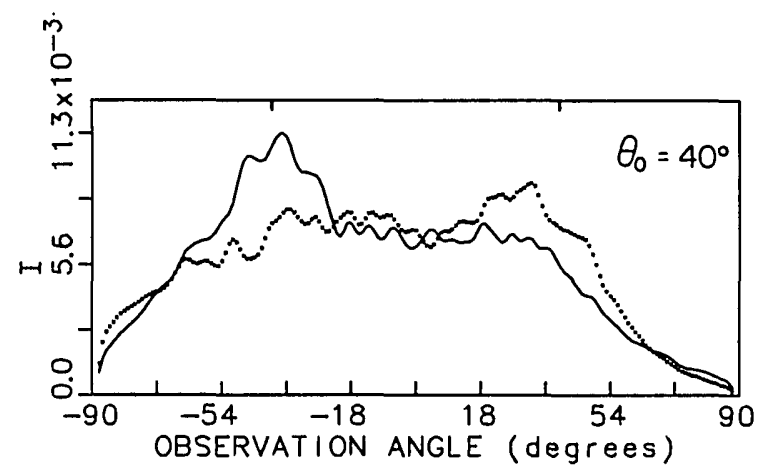

(d)

Fig. 7. Same as Fig. 2 for $T=\lambda$ and $\sigma=\lambda$. (a) $\theta_{0}=0^{\circ}$, (b) $\theta_{0}=10^{\circ}$, (c) $\theta_{0}=20^{\circ}$, (d) $\theta_{0}=40^{\circ}$. ters studied here is in clear contrast with the experimental results of Ref. 42 for $\theta_{0}<20^{\circ}$ for surfaces with $T \gg \lambda$ and $\sigma \ll$ $T$, which show no appreciable changes in the backscattering values as $\lambda$ varies. From our figures we can also conclude that the decrease in the backscattering peak with $\theta_{0}$ is greater for $p$ waves than for $s$ waves when $T \geq 0.5$ and $\sigma$ is comparable with $T$, although for smaller $T$, i.e., $T=0.2 \lambda$ [Figs. 4(a) and 4(b)], the decrease in the backscattering value is sharper for the $s$ wave than for the $p$ wave (in agreement with the results of Ref. 39).

As may be expected, at a given value of $T$ the enhanced backscattering peak increases as $\sigma$ increases. It is interesting, however, that, as $\sigma$ increases from small values at fixed $T$, the distribution of scattered intensity becomes broader and then reaches a Lambertian-like regime; then, as $\sigma$ increases further, the enhanced backscattering peak appears.
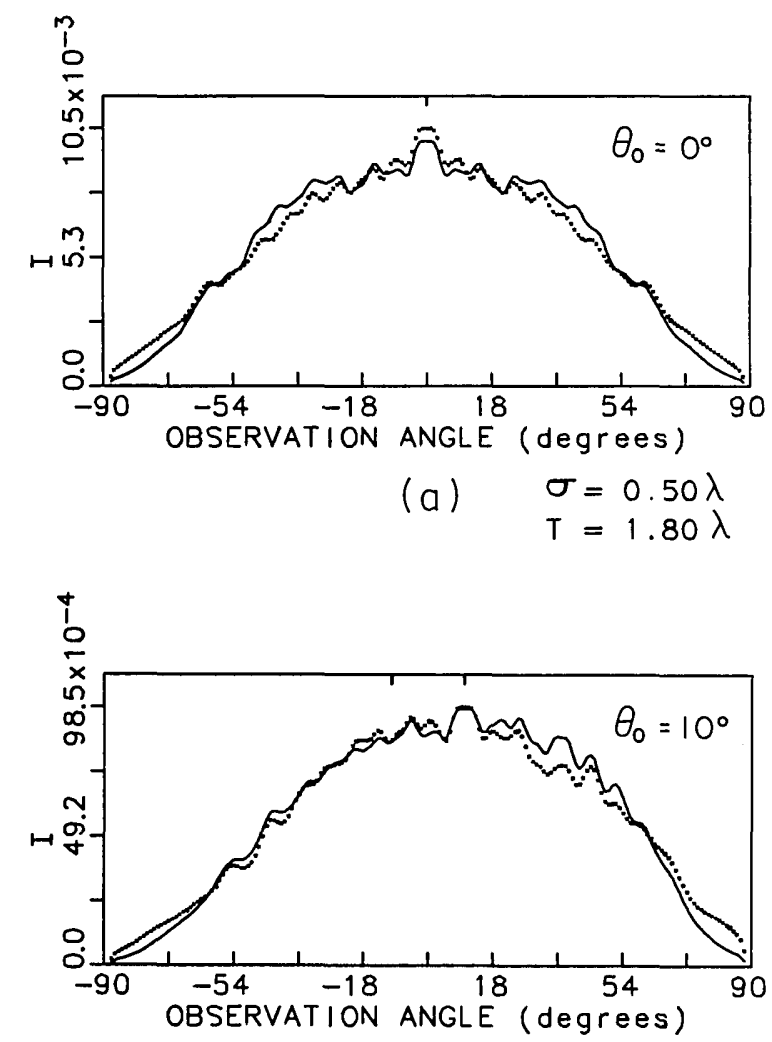

(b)

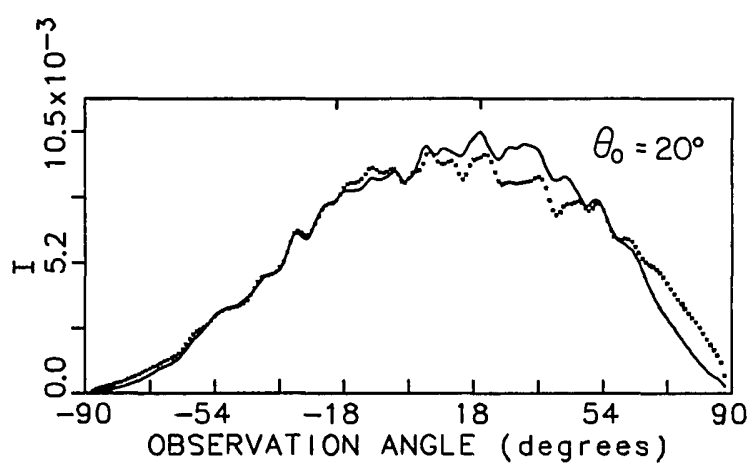

(c)

Fig. 8. Same as Fig. 2 for $T=0.5 \lambda$ and $\sigma=1.8 \lambda$. (a) $\theta_{0}=0^{\circ}$, (b) $\theta_{0}$ $=10^{\circ}$, (c) $\theta_{0}=20^{\circ}$. 


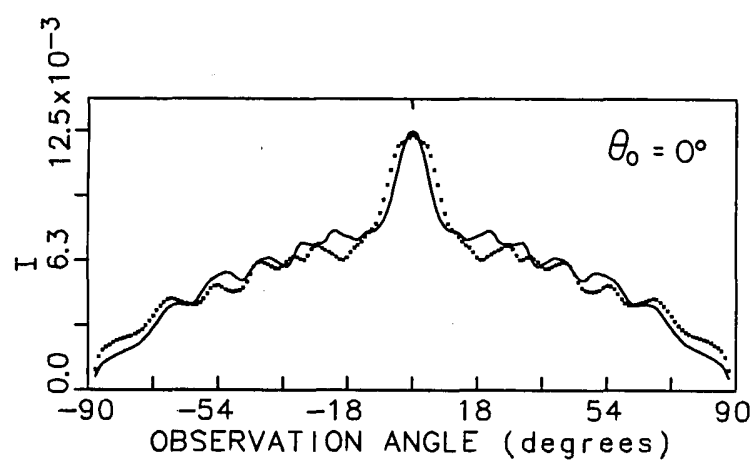

(a) $\quad \begin{aligned} & \sigma=1.00 \lambda \\ & T=1.80 \lambda\end{aligned}$

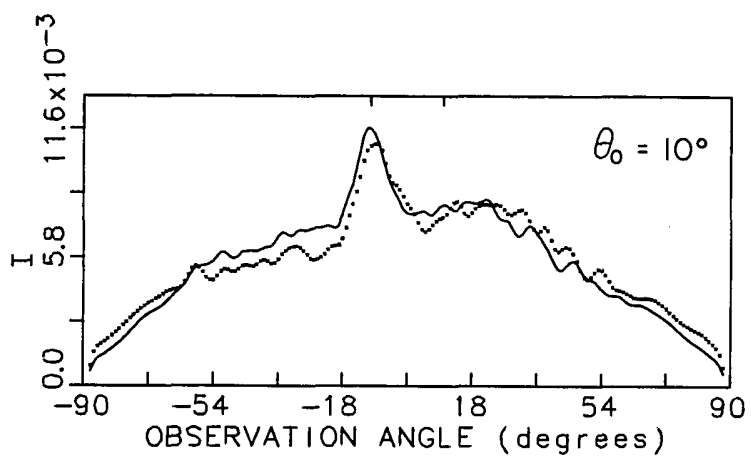

(b)

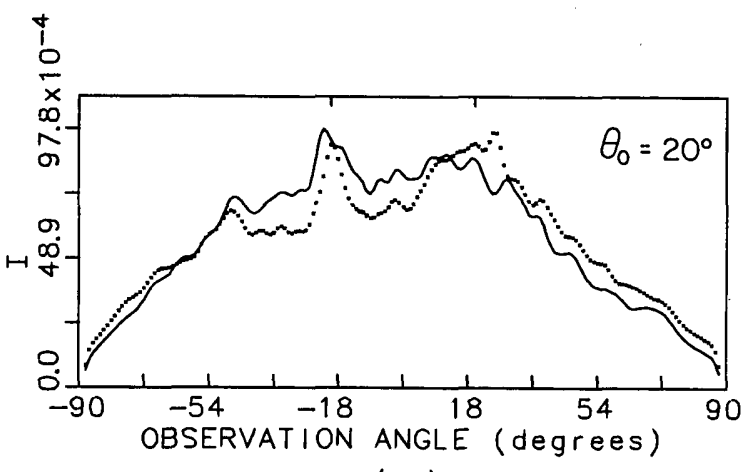

(c)

Fig. 9. Same as Fig. 2 for $T=1.8 \lambda$ and $\sigma=\lambda$. (a) $\theta_{0}=0^{\circ}$, (b) $\theta_{0}=$ $10^{\circ}$, (c) $\theta_{0}=20^{\circ}$.

It seems therefore that the Lambertian regime is a situation, as $\sigma$ increases at fixed $T$, before the regime in which the surface produces enhanced backscattering. This Lambertian characteristic is kept approximately over a relatively wide range of scattering angles and holds reasonably with incidence angles $\theta_{0}, 0^{\circ} \leq \theta_{0} \leq 20^{\circ}$. Figures $8(\mathrm{a}), 8(\mathrm{~b})$, and $8(\mathrm{c})$, for $T=1.8 \lambda$ and $\sigma=0.5 \lambda$, show an almost Lambertian distribution of mean scattered intensity over a wide interval of scattering angles and for $\theta_{0}=0^{\circ}, 10^{\circ}, 20^{\circ}$ for both $s$ and $p$ waves. Of course, as $\theta_{0}$ increases, the range of $\theta$ over which the scattering is approximately Lambertian decreases. On the other hand, Figs. 9(a), 9(b), and 9(c) show the mean scattered intensity for $T=1.8 \lambda$ and $\sigma=\lambda$, also for $\theta_{0}=0^{\circ}$, $10^{\circ}, 20^{\circ}$, confirming that for the same $T$, as $\sigma$ increases further, the enhanced backscattering appears after the Lambertian regime. Another example of Lambertian-like scat- tering is furnished by Figs. 10(a), 10(b), and 10(c), corresponding to $T=4.8 \lambda$ and $\sigma=2 \lambda$, at $\theta_{0}=0^{\circ}, 10^{\circ}, 20^{\circ}$. (Incidentally, these figures confirm once again that for sufficiently large $\sigma$ there is no difference between $s$ and $p$ scattered waves.) A comparison of Figs. 10 and 8 shows that $\sigma / T$ is similar in both cases but that the Lambertian characteristic is maintained in broader ranges of $\theta_{0}$ and $\theta$ in Fig. 10 than in Fig. 8. Thus it seems that the Lambertian behavior at fixed $\sigma / T$ is maintained over a wider range of both incidence and scattering angles as $\lambda$ decreases. It should be remarked that the appearance of the Lambertian regime as $\sigma$ increases was experimentally observed and reported in Ref. 42, although for surfaces with $T \gg \lambda$ and $\sigma \ll T$ (with $\sigma>\lambda$ ) and at large incidence angles, $50^{\circ} \leq \theta_{0} \leq 80^{\circ}$.
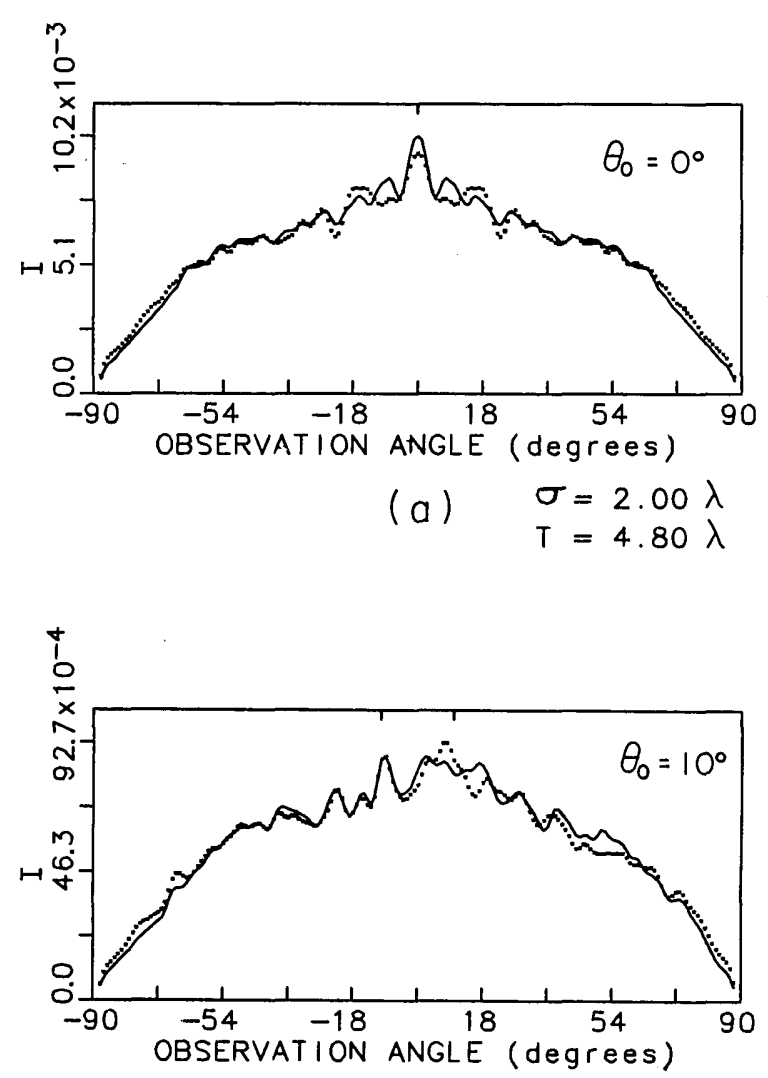

(b)

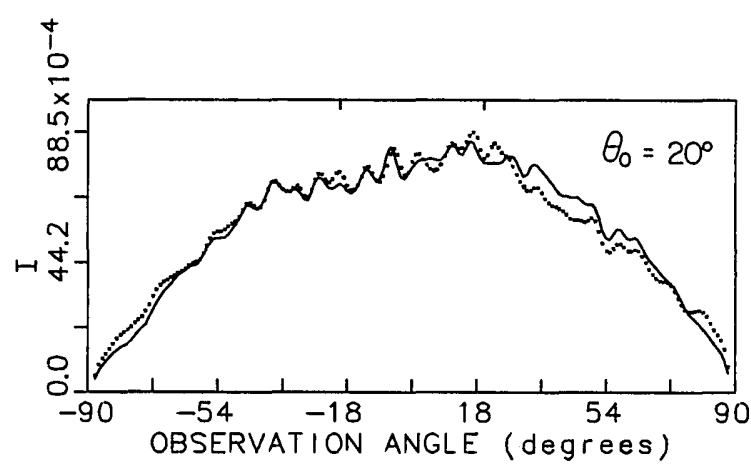

(c)

Fig. 10. Same as Fig. 2 for $T=4.8 \lambda$ and $\sigma=2 \lambda$. (a) $\theta_{0}=0^{\circ}$, (b) $\theta_{0}=$ $10^{\circ}$, (c) $\theta_{0}=20^{\circ}$. 


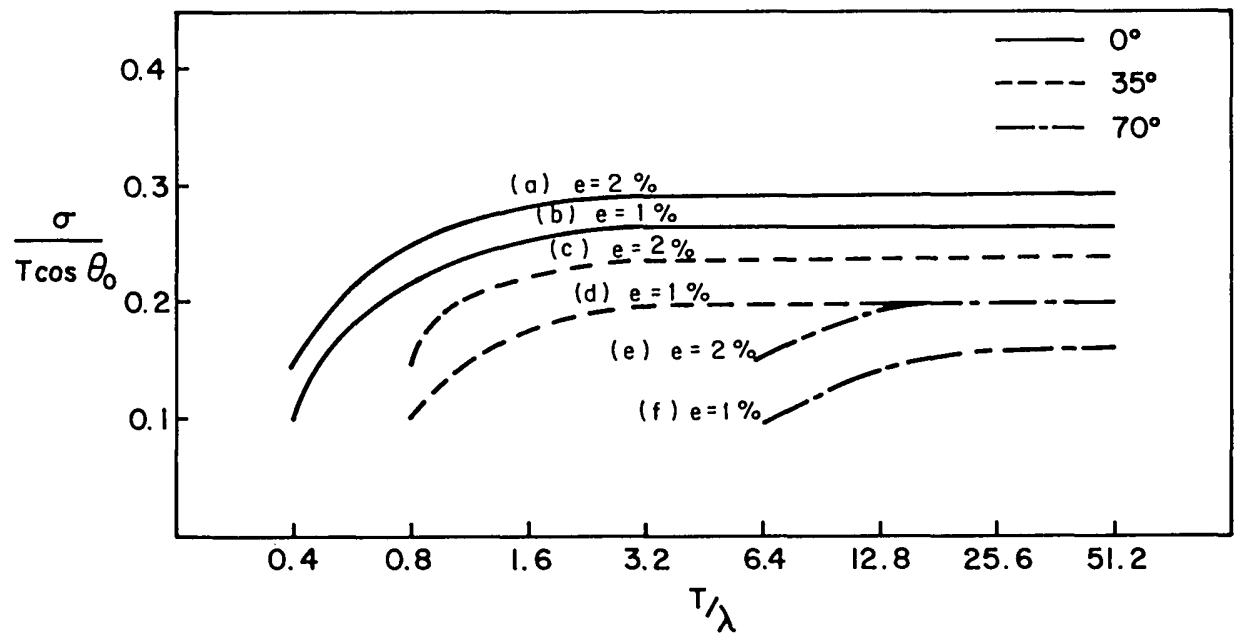

Fig. 11. Plots of $\sigma / T \cos \left(\theta_{0}\right)$ versus $T / \lambda$ at three different incidence angles: $\theta_{0}=0^{\circ}, 35^{\circ}, 70^{\circ}$, marking the zones of validity of the Kirchhoff approximation.

\section{Validity of the Kirchhoff Approximation}

The above calculations allow us to obtain criteria about the range of validity of the $\mathrm{KA}$ for which the normalized mean scattered intensity [Eq. (6)] acquires the form ${ }^{43}$

$$
\begin{aligned}
\frac{1}{I_{0}}\left\langle I_{K A}(\theta)\right\rangle=\frac{k}{\pi} & \frac{1}{\cos \theta_{0}}\left[\frac{1+\cos \left(\theta_{0}+\theta\right)}{\cos \theta_{0}+\cos \theta}\right]^{2} \\
& \times \int_{0}^{\infty} \cos \left(v_{x} \tau\right) \exp \left\{-v_{z}{ }^{2}\left[\sigma^{2}-c(\tau)\right]\right\} \mathrm{d} \tau,
\end{aligned}
$$

Where

$$
\begin{aligned}
& v_{x}=k\left[\sin \left(\theta_{0}\right)-\sin (\theta)\right], \\
& v_{z}=-k\left[\cos \left(\theta_{0}\right)+\cos (\theta)\right],
\end{aligned}
$$

and $c(\tau)$ is the height covariance given by Eq. (11).

Using the same surface statistics as in Subsection 1.C, we have performed computations with Eq. (12). A study has been made of how the resulting mean scattered intensity satisfies the unitarity condition versus $\theta_{0}$ and the surface parameters $\sigma$ and $T$. Comparisons with the exact numerical calculations of Subsection 1.B are made.

Figure 11 shows curves of $\sigma /\left(T \cos \theta_{0}\right)$ versus $T / \lambda$, for several angles of incidence, $\theta_{0}=0^{\circ}, 35^{\circ}, 70^{\circ}$, marking the frontier below which the unitarity of the KA [Eq. (12)] differs from 1 [cf. Eq. (11)] by less than 1 or $2 \%$ and above which it differs from 1 by more than 1 or $2 \%$. Thus these curves (or those similarly drawn for other angles of incidence) should mark the range within which the $\mathrm{KA}$ is approximately valid. For instance, for $T=4.8 \lambda$ and $\sigma=\lambda$ at $\theta_{0}=0^{\circ}$, there exists a point in Fig. 11 with $\sigma /\left[T \cos \left(\theta_{0}\right)\right]=0.2$, which is well down curve (b), below which the error in unitarity, $e$, is less than $1 \%$. Thus, if the KA mean scattered intensity were compared with that obtained from the ET, we would expect strong matching of the results. In Fig. 12(a) the exact mean scattered intensity is plotted, showing no differences between $s$ and $p$ polarizations, as expected; the coincidence with the KA approximation is total. Note that, in order to make the comparison easier, the KA mean scattered intensity has been plotted by two different methods: by using the smooth result of Eqs. (13) and by calculating the KA scattered field from each of 200 samples (the same samples from

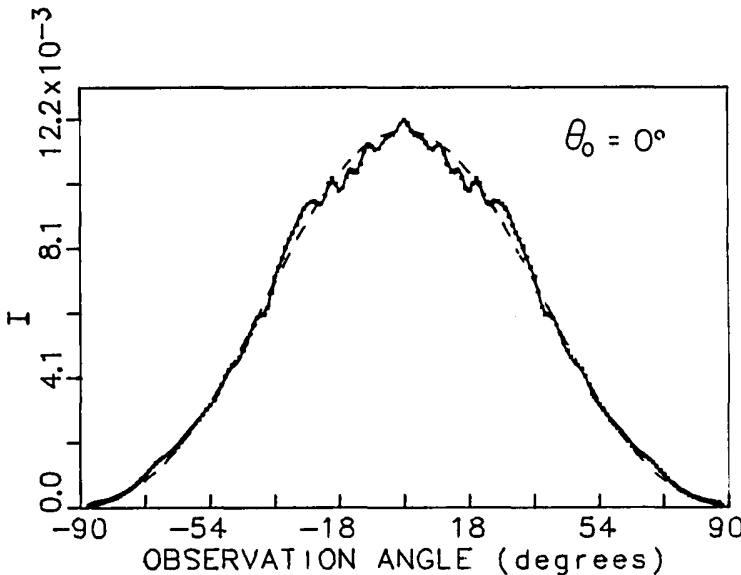

(a)

$$
\begin{aligned}
& \sigma=1.00 \lambda \\
& T=4.80 \lambda
\end{aligned}
$$

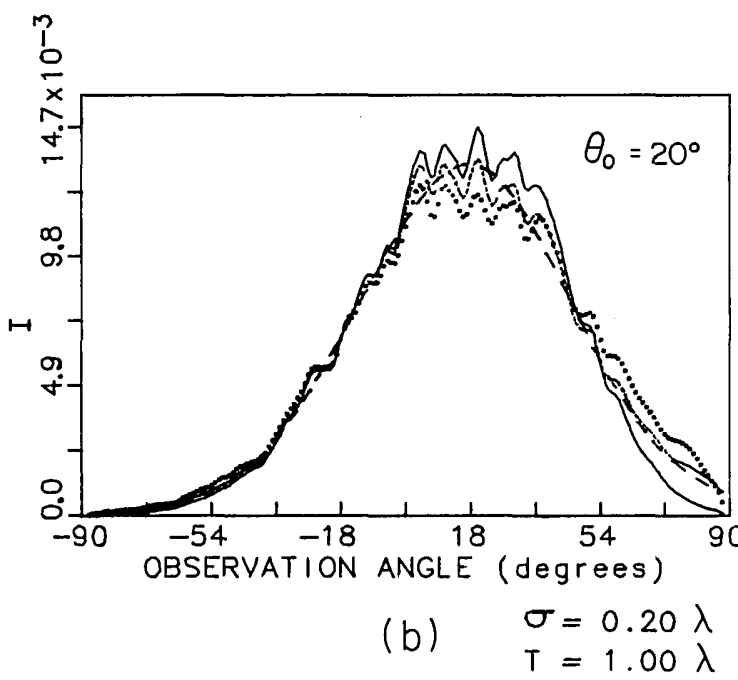

Fig. 12. (a) Same as Fig. 2 for $T=4.8 \lambda, \sigma=\lambda$, and $\theta_{0}=0^{\circ}$. Solid curves, ET result, $s$ polarization; dotted curves, ET result, $p$ polarization; short-dashed curves, KA averaging over 200 samples; longdashed curves, KA from Eq. (12). (b) Same as (a) for $T=\lambda, \sigma=$ $0.2 \lambda$, and $\theta_{0}=20^{\circ}$. 
which the ET numerical intensities were obtained) and then averaging. If the second procedure is used, the random fluctuations of the averaged KA intensity are exactly coincident with those fluctuations of the numerical ET intensity when the KA is valid, as in this example.

If we proceed to larger angles of incidence for this surface, we observe good matching of the KA and ET intensities up to $\theta_{0}=30^{\circ}$; at this $\theta$, we have $\sigma /\left(T \cos \theta_{0}\right)=0.27$, which, together with $T / \lambda=4.8$, corresponds to a point in Fig. 11 that is in curve (c), that is, with an error of approximately $2 \%$ at $\theta_{0}=30^{\circ}$.

As another example, Fig. 12(b) shows a comparison of the $\mathrm{KA}$ and ET intensities for $T=\lambda, \sigma=0.2 \lambda$, and $\theta_{0}=20^{\circ}[\sigma /(T$ $\left.\cos \theta_{0}\right)=0.21$ ]. This corresponds to a point between curves (b) and (c) of Fig. 11 for which Eq. (12) has an error $e=1.3 \%$ in the unitarity condition. In Fig. 12(b) the solid and dotted curves show the ET intensities for $s$ and $p$ polarizations, respectively, and the short- and long-dashed curves show the KA results obtained from an average over 200 samples and from Eqs. (13), respectively. As shown in the figure, the discrepancy exists, although it is small. On the other hand, at $\theta_{0}=0^{\circ}\left[\sigma / T \cos \left(\theta_{0}\right)=0.2\right]$, the corresponding point in Fig. 11 is below curve (b) and (although not shown here) shows a strong matching between the KA and ET intensities.

From the curves drawn in Fig. 11 we can see that the KA may be valid even for $T<\lambda$, provided that $\sigma /\left[T \cos \left(\theta_{0}\right)\right]$ is small. Of course, the valid $\sigma /\left[T \cos \left(\theta_{0}\right)\right]$ quickly decreases for $T<\lambda$ if the KA is valid, and, as expected, the lower $\theta_{0}$ is, the larger $\sigma /\left[T \cos \left(\theta_{0}\right)\right]$ can be for $T / \lambda$ fixed within the range of validity of the KA. From a certain value of $T / \lambda$ onward, the value of $\sigma /\left[T \cos \left(\theta_{0}\right)\right]$ below which the $\mathrm{KA}$ is valid keeps constant. For instance, at $\theta_{0}=0^{\circ}$ and for $T \geq 2$, the KA is valid for $\sigma /\left(T \cos \theta_{0}\right)$ values lower than 0.26 ; whereas, for $\theta_{0}=$ $35^{\circ}$, the validity holds for $T \geq 3 \lambda$ and $\sigma /\left(T \cos \theta_{0}\right)$ lower than 0.20 . For $\theta_{0}=70^{\circ}$ the validity of the KA is satisfied for $T \geq$ $14 \lambda$ and for $\sigma /\left(T \cos \theta_{0}\right)$ values lower than 0.2 . In this connection, the criterion $\sigma / T \cos \theta_{0}<0.2$ proposed in Ref. 27 may be considered an overall criterion that contains less detail than Fig. 11.

For $T / \lambda$ values lower than those plotted in Fig. 11 for the corresponding $\theta_{0}$, the specular peak contains most of the reflected energy, and thus the unitarity criterion used here is no longer appropriate.

Finally, it should be pointed out that the agreement between the KA and the results of experiments for $T \approx 2 \lambda, \sigma=$ $0.2 \lambda$, and $\theta_{0}=20^{\circ}\left[\sigma /\left(T \cos \theta_{0}\right)=0.11\right]$ was illustrated in Fig. 6 of Ref. 20. This agreement is obvious in Fig. 11.

\section{PERIODIC ROUGH SURFACES (REFLECTION GRATINGS)}

\section{A. Enhancement of Diffraction Orders}

A method of calculation has been established for dealing with diffraction of either $s$ - or $p$-polarized waves from deep, perfectly reflecting gratings. The essentials are presented in Appendix B. In Refs. 37 and 44 it was shown, by using this method, that both sinusoidal profiles and profiles containing few Fourier coefficients may produce (with appropriate combinations of period $a$ and amplitudes) an enhancement of either the specular order or all antispecular orders. We show here that this enhancement is quite a general phenomenon for any shape and any number of diffraction orders and is possible for large $n$, a fact of practical interest in applications; the enhancement is also intimately connected with enhanced backscattering from random surfaces.

We wish to remark, first, that the aforementioned calculations are computationally expensive. The number of terms required in Eq. (B11) or (B12) below is usually more than 30 and could even be 80 if good unitarity is required. The computation time may be reduced by a factor of 200 , however, if an approximate method is used instead. This method consists of using Eqs. (A15) and (A18) below for calculating the current densities, with the $x$ integrals extended over a few periods (typically 3 or 5 , depending on the size of the period $a$, so that edge effects are avoided as much as possible). This is equivalent to taking just the $l=0$ term in Eqs. (B11) and (B12) and integrating over several periods. The $x$ interval of integration must be chosen with its ends $x=0$ and $x=L$ coincident with points $x$ at which $D(x)$ has a peak value. (It is intuitively clear that this choice of the interval of integration can account more easily for multiple reflections than can any other choice. The numerical results confirm that it gives the closest agreement with the exact calculation.) Although it is less accurate (as manifested by the worse unitarity and hence the deviations from the exact method of calculation), this procedure is worthwhile in many cases, even though, in order to minimize the loss of accuracy, we are limited to dealing with shallower gratings than those that we could consider by using the exact calculation. (It should be remarked in this connection that a similarity between the diffraction pattern obtained by consider-
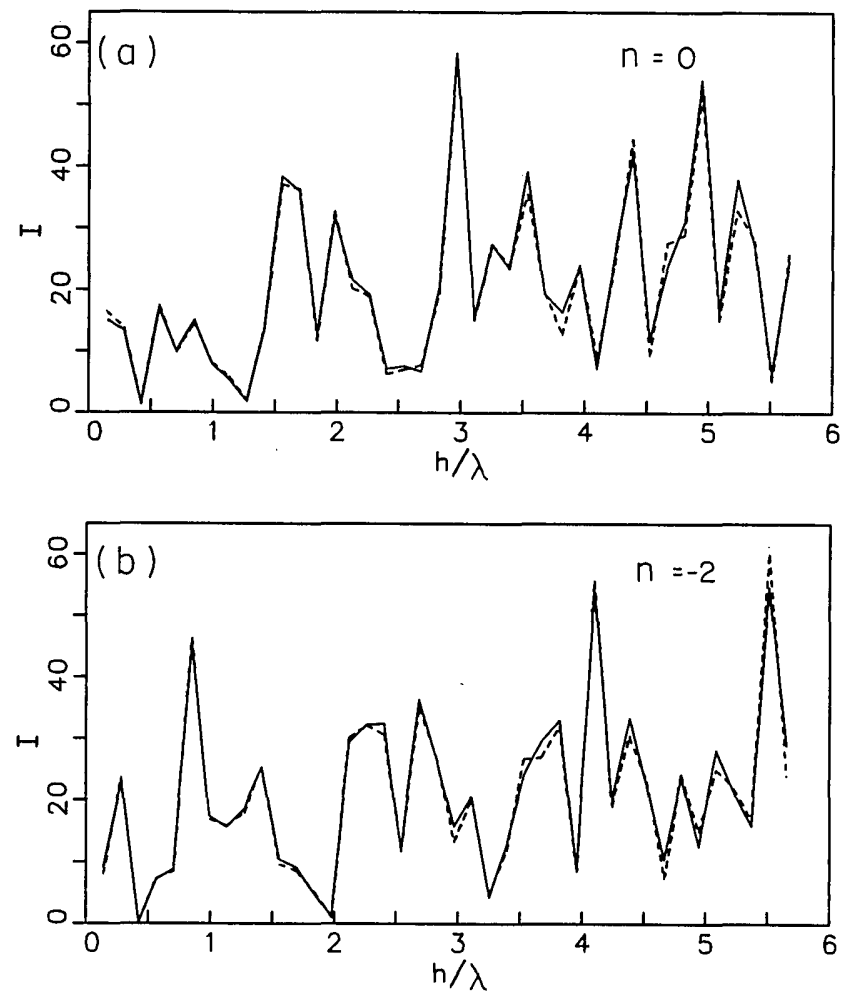

Fig. 13. (a) Specular and (b) antispecular intensities for $s$ waves versus $h / \lambda$ from $z=h \cos (2 \pi x / a)$, with $a=4.44 \lambda$ and $\theta_{0}=13^{\circ}$. Shown are exact results (solid curves) and results of the approximate calculation (dashed curves) as described in text. 


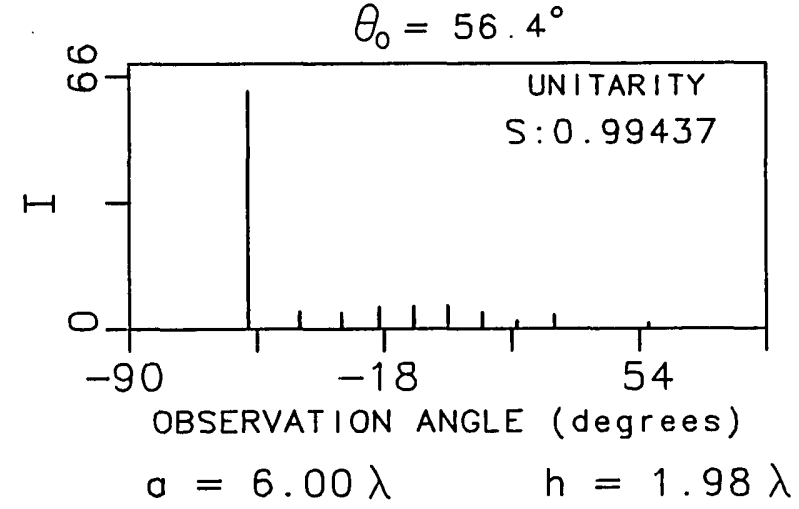

Fig. 14. Diffracted intensities of $s$ waves from sinusoidal grating with $a=6 \lambda, h=1.98 \lambda$, and $\theta_{0}=56.4^{\circ}$, showing approximately $60 \%$ enhancement at $n=-10$.

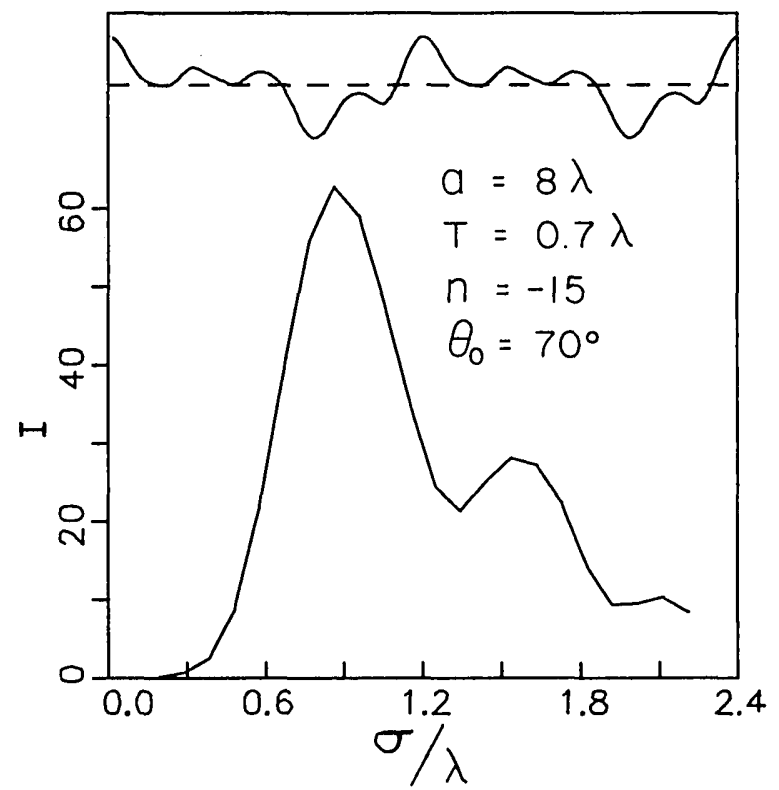

Fig. 15. Diffracted intensities of $s$ waves $n=-15$ versus $\sigma / \lambda$ for the profile at the top: $a=8 \lambda, \theta_{0}=70^{\circ}$. The random series from which this profile has been simulated has $T=0.7 \lambda$.

ing just a few periods and that obtained from an infinitely extended grating was pointed out previously, ${ }^{45}$ although this result was obtained for shallow gratings only.)

For example, for a sinusoidal grating with a profile $D(x)=$ $h \cos (2 \pi x / a)$, with $a=4.44 \lambda$, the approximate result obtained for $s$ waves, as described above, for the diffracted orders versus $h$ is similar to the exact result shown in Fig. 7 of Ref. 37. Figure 13 shows that the specular and antispecular ( $n=-2)$ results for both methods display strong similarity even at large $h$. Observe the characteristic oscillation of the orders with $h$ (note that, in the way in which we define $h$, the depth of the grating is actually $2 h$ ). All subsequent calculations, unless it is stated explicitly otherwise, are made by this approximate method, based on the fact that, whenever we have checked the approximate results with the exact ones, we have obtained good agreement.

The antispecular enhancement may be strong for high orders even if the grating supports many propagating orders and has any shape. Figure 14 shows an exact calculation of the diffraction orders for $s$ waves (for $p$ waves the result is practically identical and is not shown here) for a sinusoidal grating with $a=6 \lambda$ and $h=2 \lambda$ at $\theta_{0}=56.4^{\circ}$, showing an enhancement of approximately $60 \%$ of the diffracted energy in the antispecular order $n=-10$. A variation in $h$ of approximately $10 \%$ from this value does not produce a significant change in this result. With arbitrary profiles it can also be obtained easily. Figure 15 depicts the variation of the antispecular order $n=-15$ with $\sigma$ for the arbitrary grating profile shown. The period is $a=8 \lambda$. This profile $D(x)$ has been simulated from an infinite random series with adjustable variance (ranging from $\sigma=0.1 \lambda$ to $\sigma=2.5 \lambda$ ). If we take from this series a portion of length $a=8 \lambda$ and then periodically repeat it, the resulting grating has many Fourier coefficients. The enhancement of the antispecular order $n$ $=-15$ is obtained at $\theta_{0}=70^{\circ}$ and for $\sigma=0.9 \lambda$, showing an intensity of $65 \%$ of the total reflected energy. As shown in Fig. 15, the variation in this order with $\sigma$ oscillates with a large period, since the incidence angle is large. This slow oscillation with $\sigma$ at large $\theta_{0}$ (and hence at large orders) was pointed out before and permits deviations in $\sigma$ that still give large enhancement with respect to the value at which it is maximum.

The two examples just presented illustrate some of the many possibilities of designing gratings with blaze in a high order ( $n=-10,-15$, or even higher) in Littrow mounting and show that this effect can be obtained for any profile and for any $a$, even though, if $a$ is large, the existence of many propagating orders makes the blaze much lower than $100 \%$. It can be increased further by making $h$ (or $\sigma$ ) even larger. (In a sinusoidal grating the mean height is $\sigma=h / \sqrt{2}$.)

In fact, at large $\theta_{0}$, the high antispecular order progressively increases, with the possibility of oscillation but with an increasing envelope, thus showing that we must consider large values for $h$ (or $\sigma$ ) to see a large enhancement of this order in Littrow mounting. Also, the $h$ (or $\sigma$ ) value at which the order starts increasing is greater the larger is the period $a$. This is illustrated in Fig. 16, in which the behavior with $h$

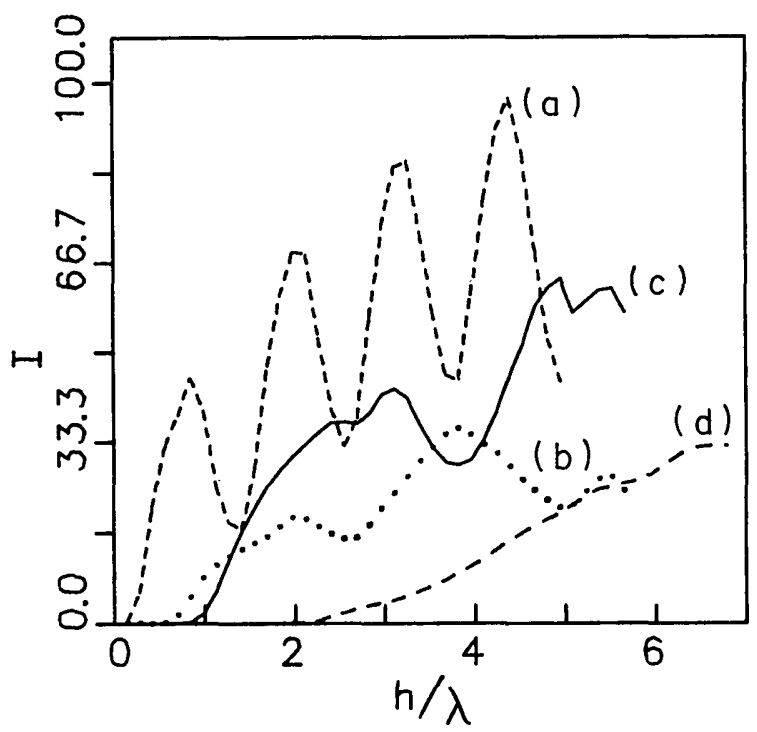

Fig. 16. Diffracted intensities of $s$ waves from sinusoidal gratings versus $h / \lambda$. Curve (a), $a=1.78 \lambda, \theta_{0}=57.6^{\circ}, n=-3$; curve (b), $a=$

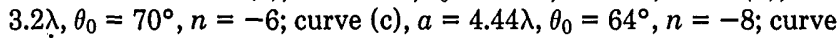
(d), $a=8 \lambda, \theta_{0}=70^{\circ}, n=-15$. 


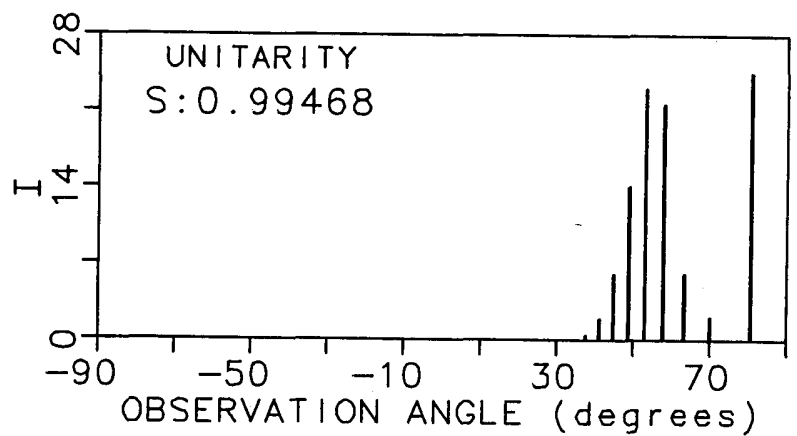

(a)

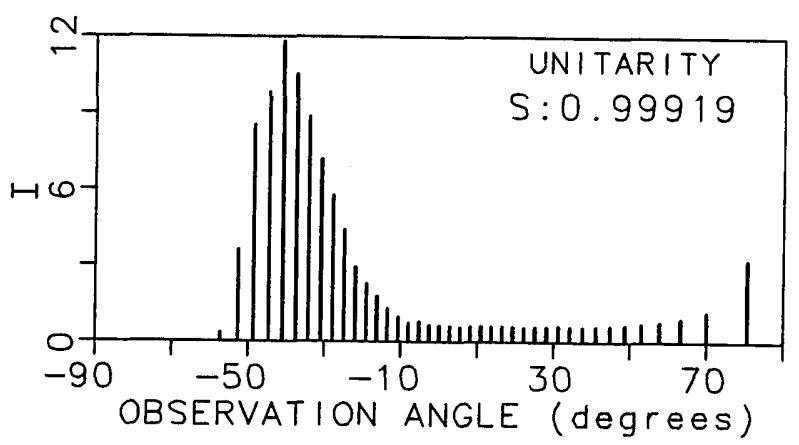

(b)

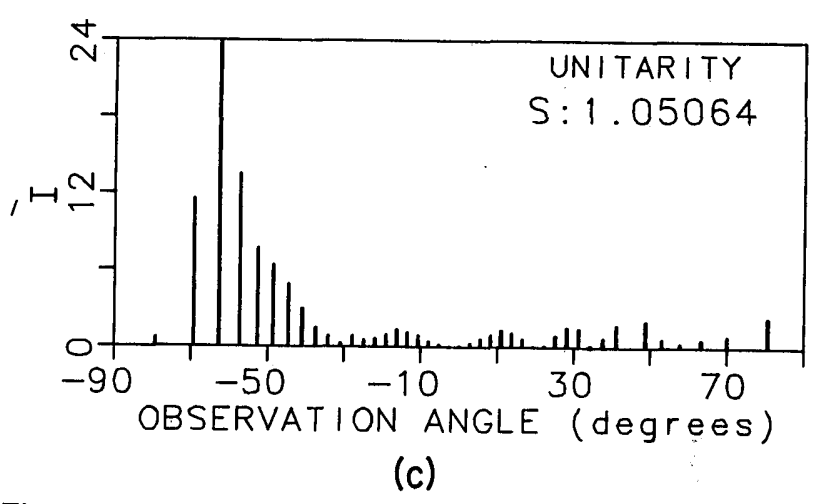

Fig. 17. Diffracted intensities of $s$ waves from a sinusoidal grating with $a=21.33 \lambda$ and $\theta_{0}=70^{\circ}$. (a) $h=0.71 \lambda$, (b) $h=5.66 \lambda$, (c) $h=$ $11.31 \lambda$.

of high- $n$ antispecular orders for $s$ polarization is shown for sinusoidal gratings with periods: $a=1.78 \lambda\left(\theta_{0}=57.6^{\circ}, n=\right.$ $-3), a=3.2 \lambda\left(\theta_{0}=70^{\circ}, n=-6\right), a=4.4 \lambda\left(\theta_{0}=64^{\circ}, n=-8\right)$, and $a=8 \lambda\left(\theta_{0}=70^{\circ}, n=-15\right)$. Although not shown here, the specular order has the opposite behavior [cf. Figs. 4(a) and 7 of Ref. 37]. Therefore we would expect that, when $\theta_{0}$ is large and the period is large enough to support many propagating orders, these would tend to gather nearer the antispecular direction as the grating becomes deeper, without the fluctuations observed at lower $\theta_{0}$ (cf. Refs. 37 and 44). Figures 17(a), 17(b), and 17(c) confirm this fact for a sinusoidal grating with $a=21.3 \lambda$ and $h=0.71 \lambda, 5.65 \lambda, 11.3 \lambda$, respectively (remember that the depth is $2 h$ ), at $\theta_{0}=70^{\circ}$. The calculations used in these figures are exact and have been made for both $s$ and $p$ polarizations. We show the results only for $s$ waves; however, the $p$ waves are practically identical: this happens in Fig. 7(a) because the KA is valid (we have observed the KA to be valid for sinusoidal gratings within criteria similar to those for random gratings). The results for the $p$ waves are, on the other hand, similar to those for the $s$ waves in Figs. 17(b) and 17(c) because $h$ is large in these cases. As shown in these figures, the bulk of diffracted orders progressively shifts toward the antispecular direction as $h$ increases. For such a large period as in this case, the amplitude $h$ should be increased further to obtain the stronger order exactly in the antispecular. However, the

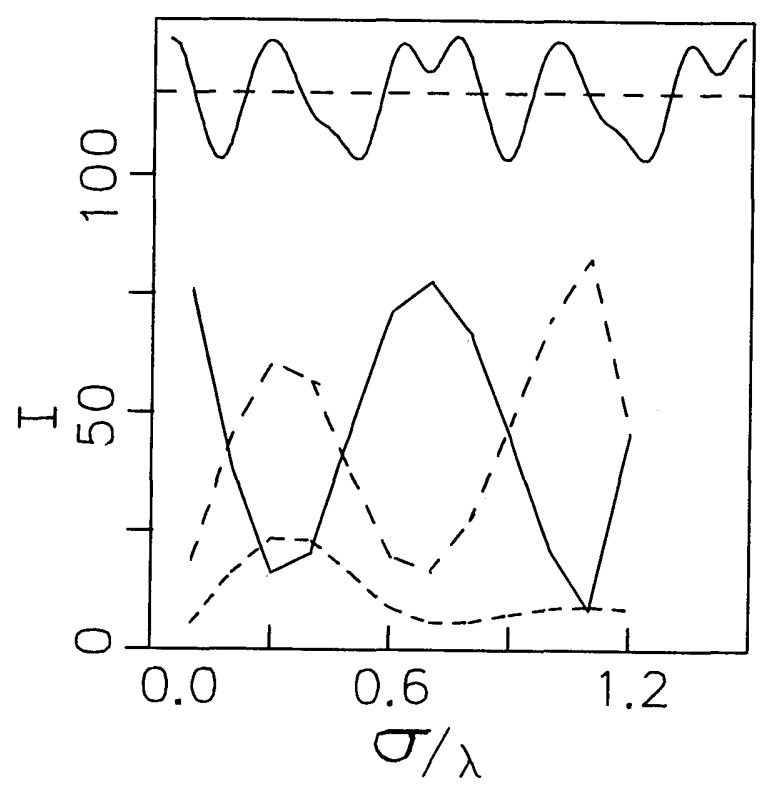

(a)

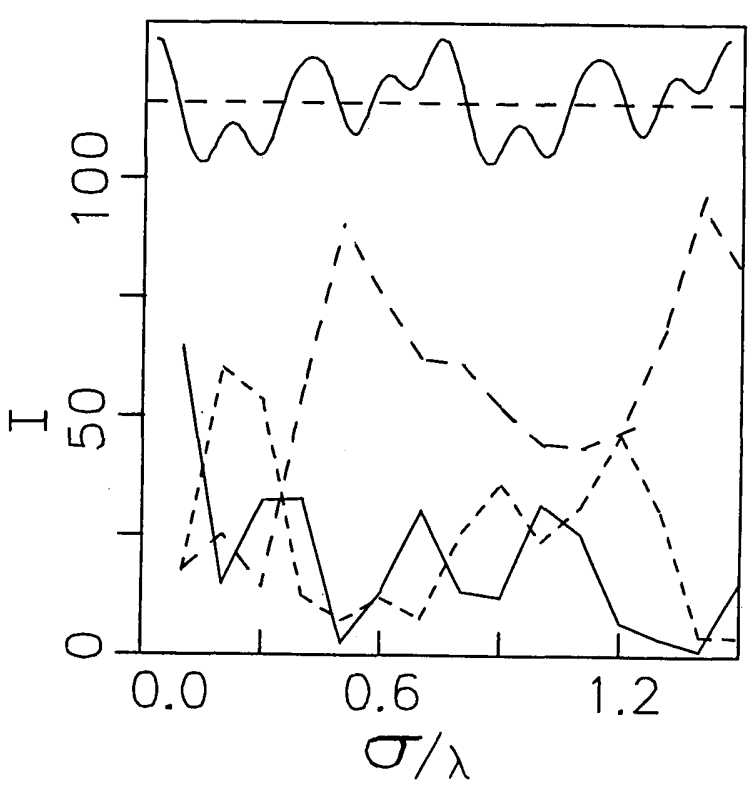

(b)

Fig. 18. Diffracted intensities of $s$ waves for $\theta_{0}=34^{\circ}$ versus $\sigma / \lambda$ for two gratings (a) and (b) with a period $a=1.78 \lambda$ and with a profile shown at the top, extracted from two different portions of the same series with $T=0.24 \lambda$. Solid curves, specular order; long-dashed curves, antispecular order $(n=-2)$; short-dashed curves, order $n=$ -1 . 


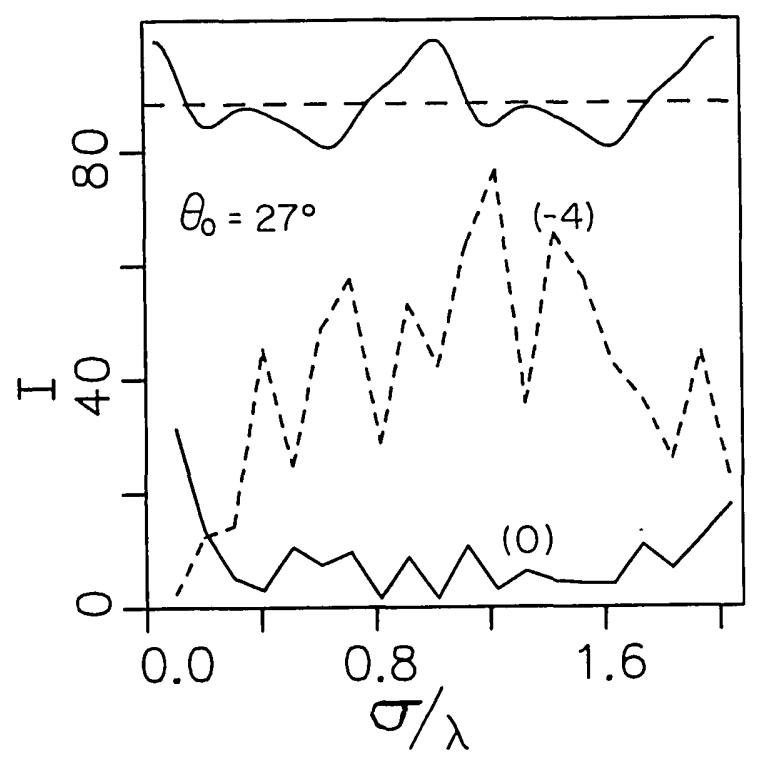

Fig. 19. Same as Fig. 18 for grating profile shown at the top, with $\theta_{0}$ $=27^{\circ}, a=4.44 \lambda$; and $T=0.7 \lambda$. Short-dashed curve, antispecular order $(n=-4)$; solid curves, specular order.
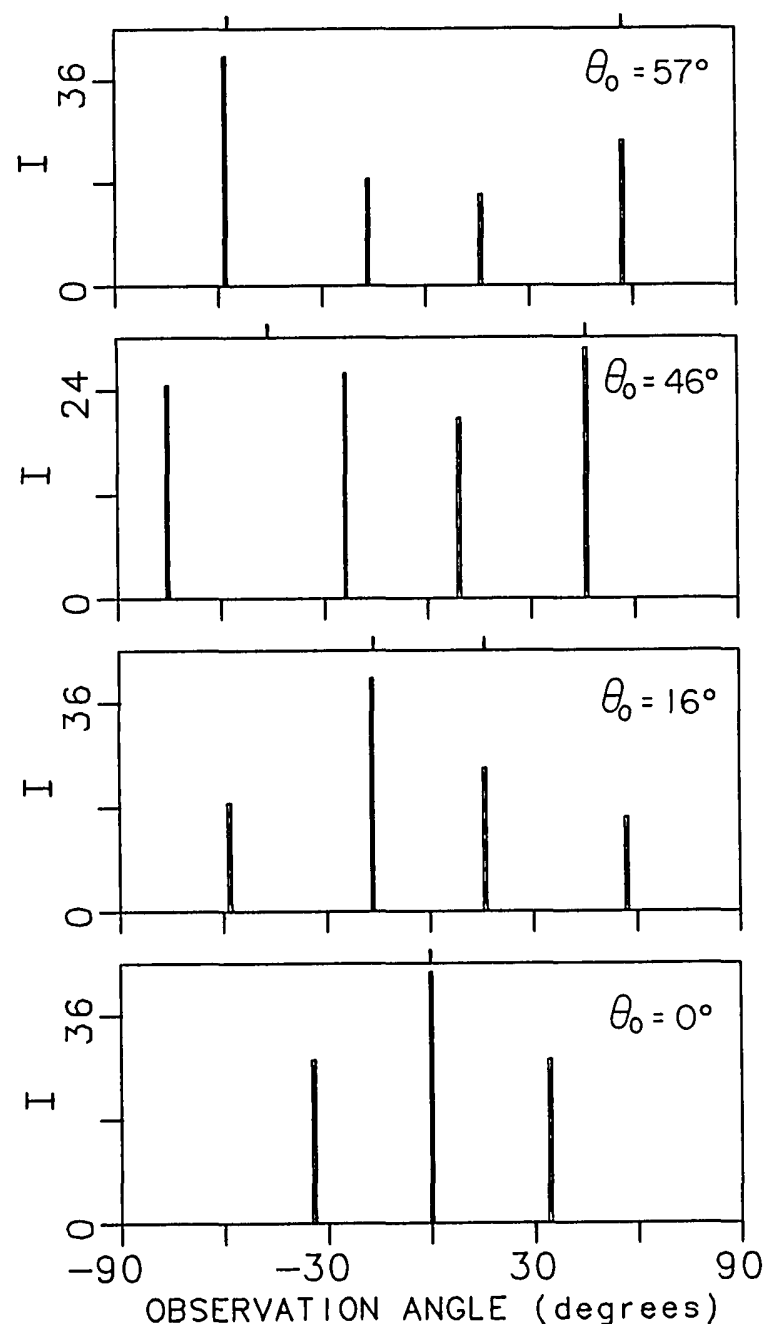

Fig. 20. Averaged diffracted intensities of $s$ waves from 200 profiles with $T=0.24 \lambda, \sigma=0.5 \lambda$, and $a=1.78 \lambda$, for $\theta_{0}=0^{\circ}, 16^{\circ}, 46^{\circ}$, $57^{\circ}$. calculation beyond $h=11.3 \lambda$ starts to lose accuracy. The figures suggest, however, that at larger $h$ an enhanced antispecular order would be expected.

\section{B. Connection with Enhanced Backscattering from Random Surfaces}

It has been shown that the enhancement of a diffraction order, in particular, the antispecular order, can be obtained for any shape and regardless of how large the period is. It can even occur for the antispecular orders in gratings with large period and under great incidence angles $\theta_{0}$. In Refs. 37 and 44 it was shown that, for deep sinusoidal gratings and also for those with a few Fourier coefficients, the enhancement takes place predominantly either in the specular order or in the antispecular order. Of course, a special profile could be devised with enhancement in some particular order different from the specular or antispecular; however, if a deep profile is indicated $a$ priori, then either the antispecular or the specular order has the highest probability of being enhanced. If the profile over one period is not symmetric, then the antispecular order has an even higher probability than the specular of being enhanced. This result suggests that this effect may occur for any shape, even if we construct $D(x)$ by periodically repeating, with period $a$, an interval of a deep random series with $\sigma$ and $T$ given. If $a$ became large, we would then get a supergrating with a random profile

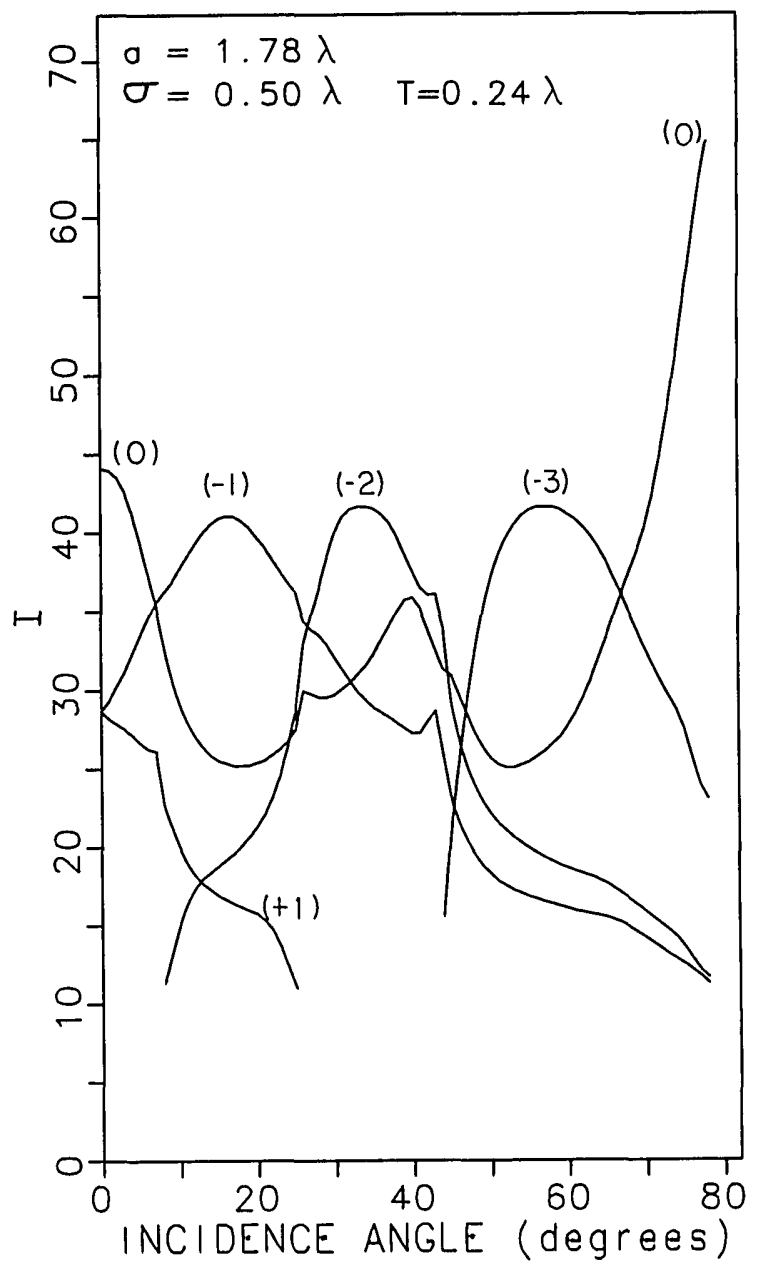

Fig. 21. Mean efficiencies versus $\theta_{0}$ for the same gratings as in Fig. 20. 

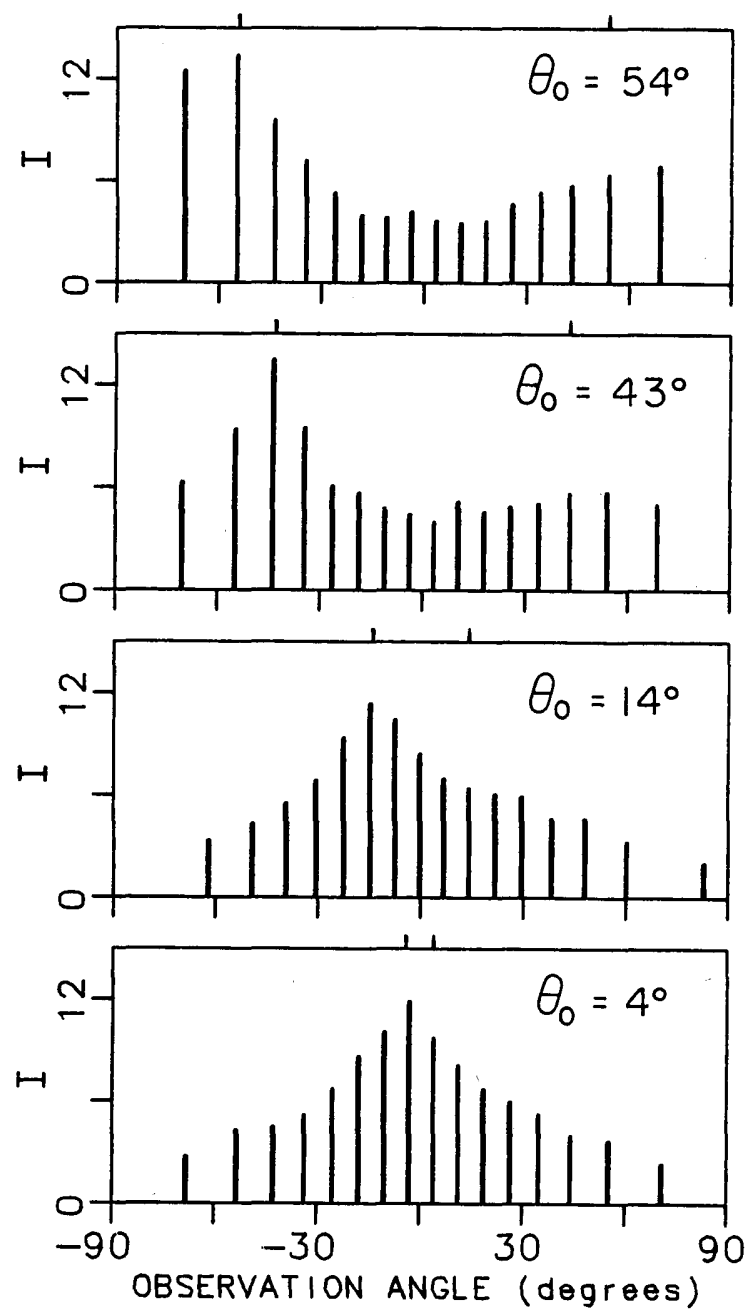

Fig. 22. Same as Fig. 20 for $T=0.7 \lambda, \sigma=1.2 \lambda$, and $a=8 \lambda, \theta_{0}=4^{\circ}$, $14^{\circ}, 43^{\circ}, 54^{\circ}$.

inside the period. According to the above results, the diffracted intensity will probably present either an enhanced specular order or an enhanced antispecular order. For each particular profile anything can happen. However, we may expect that if we average over many diffracted intensities and if each intensity results from a supergrating obtained by choosing a different interval of the random series, then the enhancement of the antispecular order will result if the profiles are not symmetric over one period. In what follows we illustrate this process.

Figure 18(a) shows for $s$ polarization the orders $0,-1$, and -2 (antispecular) at $\theta_{0}=34^{\circ}$ from a grating of period $a=$ $1.78 \lambda$ whose shape (enclosed) corresponds to a portion of a random profile with $T=0.24 \lambda$ and $\sigma$ varying from $0.1 \lambda$ to

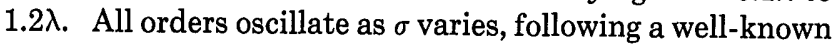
rainbow pattern, ${ }^{30,46}$ and most of the diffracted energy goes either to the specular order or to the antispecular order. A similar behavior takes place for other incidence angles $\theta_{0}$ (at high $\theta_{0}$ the oscillation period becomes large). Figure 18(b) shows the results for another profile with the same values of $T$ and $a$ as in Fig. 18(a). Now the antispecular order, and even the $n=-1$ order, is stronger than the specular order. Figure 19 plots the antispecular $(-4)$ and specular orders under $\theta_{0}=27^{\circ}$ for a grating with $a=4.44 \lambda$ simulated from a random series with $T=0.7 \lambda$. Here, again, the antispecular order is dominant.

The above examples show some of the diverse possible situations for one particular profile. In fact, it is known that the enhanced backscattering is generally difficult to observe from just one random sample, since it can appear to be swamped in the speckle fluctuations of the intensity distribution. The same difficulty may occur with the intensity diffracted from these random gratings. For one particular grating it may be that the antispecular order is not the stronger one. However, from all the above results, we have reasons to believe that the nonspecular order will dominate on the average. This is shown in what follows.

Figure 20 shows the average of the diffracted intensities at $\theta_{0}=0^{\circ}, 16^{\circ}, 46^{\circ}, 57^{\circ}$, each obtained from a grating with $a=$ $1.78 \lambda$, corresponding to a different portion of an infinite random series with $T=0.24 \lambda$ and $\sigma=0.5 \lambda$. The average has been made over 200 gratings. The result is practically the same for both $s$ and $p$ polarizations. As can be seen, in this average the antispecular order is the larger one whenever it exists. Figure 21 shows the variation of the average of each diffracted order with $\theta_{0}$, the maximum of each order exactly at that angle at which it is antispecular. Between the peaks the specular order can grow and be dominant, such as nearly $\theta_{0}=46^{\circ}$, and of course, the specular order is dominant at large $\theta_{0}$.

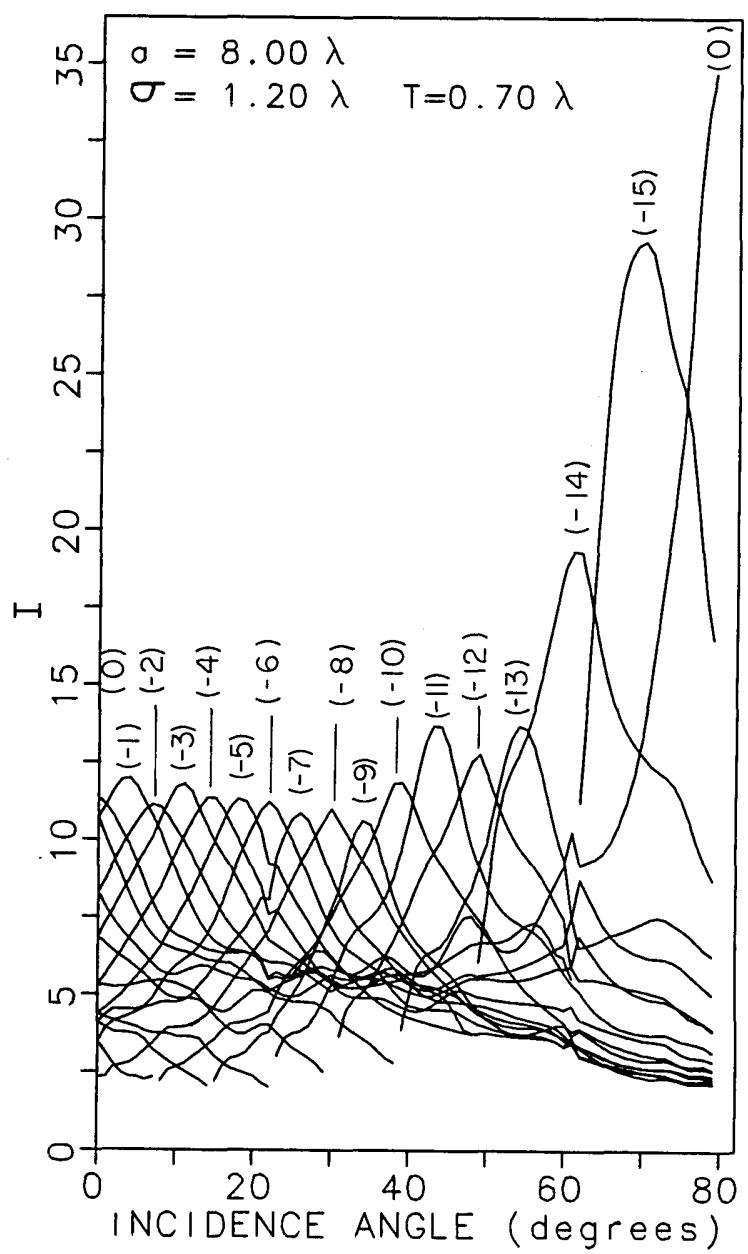

Fig. 23. Mean efficiencies versus $\theta_{0}$ for the same gratings as in Fig. 22. 
When the period increases, there exist more orders, and then for almost any incidence angle there exists an antispecular order, which is dominant. Figure 22 shows the diffracted intensities for $\theta_{0}=4^{\circ}, 14^{\circ}, 43^{\circ}, 54^{\circ}$ (no appreciable differences between $s$ and $p$ polarizations), averaged over 200 gratings with $a=8 \lambda, T=0.7 \lambda$, and $\sigma=1.2 \lambda$. The variations of these mean intensities with $\theta_{0}$ are plotted in Fig. 23, which shows the maxima exactly at the antispecular directions. The specular order cannot grow except near $\theta_{0}=$ $80^{\circ}$. When the period is infinite, the grating becomes strictly a random rough surface, and every angle of incidence has exactly an antispecular scattering direction at which the mean scattered intensity appears enhanced.

\section{CONCLUSIONS}

In this paper we have presented results based on the numerical use of the extinction theorem for one-dimensional perfectly conducting deeply rough surfaces. Two different cases were considered: random rough surfaces and reflection gratings.

For random surfaces the main results concerning the mean scattered intensity can be summarized as follows:

(1) When $\sigma / \lambda$ is small but $T / \lambda$ is also small, so that $\sigma$ is comparable with $T, s$ polarization gives a strong specular peak, in contrast to $p$ polarization, which gives a broad halo skewed toward the backscattering direction.

(2) Enhanced backscattering is predicted for one-dimensional random surfaces with moderate and high $\sigma / T$, and the backscattering peak increases as $\sigma / T$ increases.

(3) The backscattering peak appears more enhanced with respect to the background halo at an incidence angle of $\theta_{0}=20^{\circ}$. It also decreases as $\theta_{0}$ increases.

(4) Within the range $0.2 \leq T / \lambda \leq 5$, the backscattering peak has two different behaviors:

(a) At $\theta_{0}<20^{\circ}$ with $\sigma / T$ constant, the peak increases slightly as $\lambda$ decreases.

(b) At $\theta_{0}>20^{\circ}$ with $\sigma / T$ constant, the peak decreases slightly as $\lambda$ decreases.

(5) As $\sigma / \lambda$ increases from low values at fixed $T / \lambda$, there is a regime at which the mean scattered intensity follows approximately Lambert law; this is before the intensity reaches the regime at which it produces enhanced backscattering. The Lambertian distribution of mean scattered intensity is obtained approximately for $\theta_{0} \leq 20^{\circ}$, and it is maintained under a broad range of scattering angles $\theta$. This range becomes narrower as $\theta_{0}$ increases.

(6) When $\sigma / \lambda$ is larger than unity we do not observe a difference between $s$ and $p$ polarizations within the numerical accuracy that we obtain.

(7) We have established the range of validity of the KA for random surfaces and $T / \lambda>0.4$. On the whole, the KA is approximately valid for $\sigma / T \cos \theta_{0}<0.2, T>0.5 \lambda$, and $\theta_{0} \leq$ $40^{\circ}$. For larger $\theta_{0}$ the KA is still valid for larger $T$, whereas for $T / \lambda<0.4$ the ratio $\sigma / T \cos \theta_{0}$ decreases further. Precise curves marking the range of validity of the $\mathrm{KA}$ are shown in Fig. 11 for several angles of incidence.
For deep reflection gratings we have obtained results from both an exact numerical method and an approximate procedure that performs the $x$ integration over a few periods, saving a large amount of computing time. The main results are as follows:

(1) Enhancement (blaze) of the antispecular order can be obtained for any shape regardless of how large the period is, even at large incidence angles. Therefore blaze of $>50 \%$ can be obtained for high orders. For deep gratings there is no significant difference between the results for $s$ polarization and those for $p$ polarization.

(2) We can easily devise gratings that present enhancement in all antispecular orders.

(3) Although particular profiles may yield enhancement of any order that is neither specular nor antispecular, statistically these two orders are those with the highest probability of enhancement if the profile is given a priori. If the period is large and the unit cell has no symmetry, the antispecular is even more likely to be enhanced than the specular order.

(4) All orders oscillate following a rainbow pattern as the grating depth increases. These oscillations have a larger period as $\theta_{0}$ increases.

(5) The statistically predominant enhancement of the antispecular order for deep gratings with a large period and whose unit cell has no center of symmetry is connected directly with the enhanced backscattering observed in deep random surfaces. This is understood by considering the mean scattered intensity from a random surface to be the distribution obtained by averaging over many samples, each being a supergrating (namely, one with a large period, so that for any incidence angle there is an antispecular order). After the average is obtained, the antispecular appears enhanced with respect to the other orders.

Finally, we remark that when this paper was completed, Thorsos ${ }^{47}$ reported a study on the validity of the KA that used a procedure similar to ours.

\section{APPENDIX A}

Let $\left[\mathbf{E}^{(i)}(\mathbf{r}), \mathbf{H}^{(i)}(\mathbf{r})\right]$ be the electromagnetic field incident upon the scatterer; the integral equations for the interaction are as follows. ${ }^{9}$ For the electric vector, the extinction theorem yields

$$
\mathbf{E}^{(i)}\left(\mathbf{r}_{<}\right)+(1 / 4 \pi) \mathbf{S}_{e}\left(\mathbf{r}_{<}\right)=\mathbf{0}
$$

whereas in the upper half-space the scattered field satisfies

$$
\mathbf{E}^{(s)}\left(\mathbf{r}_{>}\right)=(1 / 4 \pi) \mathbf{S}_{e}\left(\mathbf{r}_{>}\right) .
$$

In Eqs. (A1) and (A2), $\mathbf{r}_{>}$and $\mathbf{r}_{<}$denote the position vectors of points outside and inside the scatterer, respectively. In the case under consideration, $\mathbf{r}_{>}$and $\mathbf{r}_{<}$correspond to points with $z>D(x)$ and $z<D(x)$, respectively.

For perfect conductors, $\mathbf{S}_{e}(\mathbf{r})$ is $^{9}$

$$
\mathbf{S}_{e}(\mathbf{r})=(4 \pi i k / c) \int_{S} \mathbf{J}(\mathbf{r}) \tilde{\mathbf{G}}\left(\mathbf{r}, \mathbf{r}^{\prime}\right) \mathrm{d} S^{\prime}
$$


for which $\mathbf{J}(\mathbf{r})$ is the induced electric current density, $\mathrm{d} S^{\prime}$ is the surface element, and $\tilde{\mathbf{G}}\left(\mathbf{r}, \mathbf{r}^{\prime}\right)$ is Green's dyadic:

$$
\tilde{\mathbf{G}}\left(\mathbf{r}, \mathbf{r}^{\prime}\right)=\left(\tilde{\mathbf{U}}+1 / k^{2} \nabla \nabla\right) G_{0}\left(\mathbf{r}, \mathbf{r}^{\prime}\right),
$$

where $G_{0}\left(\mathbf{r}, \mathbf{r}^{\prime}\right)$ is the scalar Green's function and $\tilde{\mathbf{U}}$ is the unit dyadic.

For the magnetic field, in the lower half-space we have

$$
\mathbf{H}^{(i)}\left(\mathbf{r}_{<}\right)+(1 / 4 \pi) \mathbf{S}_{h}\left(\mathbf{r}_{<}\right)=0
$$

and in the upper half-space we have

$$
\mathbf{H}_{(s)}\left(\mathbf{r}_{>}\right)=(1 / 4 \pi) \mathbf{S}_{h}\left(\mathbf{r}_{>}\right),
$$

where

$$
\mathbf{S}_{h}(\mathbf{r})=(4 \pi / c) \int_{S} \mathbf{J}\left(\mathbf{r}^{\prime}\right) \cdot \nabla \times \tilde{\mathbf{G}}\left(\mathbf{r}, \mathbf{r}^{\prime}\right) \mathrm{d} S^{\prime} .
$$

In the two-dimensional geometry associated with the onedimensional surface under consideration, $G_{0}\left(\mathbf{r}, \mathbf{r}^{\prime}\right)$ is given by the zeroth-order Hankel function of the first kind: $i \pi H_{0}{ }^{(1)}\left(k\left|\mathbf{r}-\mathbf{r}^{\prime}\right|\right)$; on the other hand, the element of surface is

$$
\mathrm{d} S^{\prime}=\left[1+\left(\frac{\mathrm{d} D}{\mathrm{~d} x^{\prime}}\right)^{2}\right]^{1 / 2} \mathrm{~d} x^{\prime}
$$

For the electric field, Eq. (A3) becomes

$$
\begin{aligned}
\mathbf{S}_{e}(\mathbf{r})=\frac{-4 \pi^{2} k}{c} \int_{-\infty}^{\infty} & \mathbf{J}\left(\mathbf{r}^{\prime}\right)\left(\tilde{\mathbf{U}}+\frac{1}{k^{2}} \nabla \nabla\right) \\
& \times H_{0}^{(1)}\left(k\left|\mathbf{r}-\mathbf{r}^{\prime}\right|\right)\left[1+\left(\frac{\mathrm{d} D}{\mathrm{~d} x^{\prime}}\right)^{2}\right]^{1 / 2} \mathrm{~d} x^{\prime},
\end{aligned}
$$

where

$$
\mathbf{r}^{\prime}=\left[x^{\prime}, z^{\prime}=D\left(x^{\prime}\right)\right] .
$$

By extracting components along $0 X, 0 Y$, and $0 Z$ from the vector Eq. (A9), we obtain

$$
\begin{aligned}
& {\left[S_{e}(\mathbf{r})\right]_{x}=-\frac{4 \pi^{2} k}{c} \int_{-\infty}^{+\infty}\left[J_{x}\left(\mathbf{r}^{\prime}\right)-J_{x}\left(\mathbf{r}^{\prime}\right) \frac{1}{k^{2}} \frac{\partial^{2}}{\partial x^{\prime 2}}\right.} \\
& \left.+\frac{J_{z}\left(\mathbf{r}^{\prime}\right)}{k^{2}} \frac{\partial}{\partial z^{\prime}} \frac{\partial}{\partial x^{\prime}}\right] H_{0}^{(1)}\left(k\left|\mathbf{r}-\mathbf{r}^{\prime}\right|\right)\left[1+\left(\frac{\mathrm{d} D}{\mathrm{~d} x^{\prime}}\right)^{2}\right]^{1 / 2} \mathrm{~d} x^{\prime}, \\
& {\left[S_{e}(\mathbf{r})\right]_{y}=-\frac{4 \pi^{2} k}{c} \int_{-\infty}^{+\infty} J_{y}\left(\mathbf{r}^{\prime}\right) H_{0}^{(1)}\left(k\left|\mathbf{r}-\mathbf{r}^{\prime}\right|\right)} \\
& {\left[S_{e}(\mathbf{r})\right]_{y}=-\frac{4 \pi^{2} k}{c} \int_{-\infty}^{+\infty}\left[J_{z}\left(\mathbf{r}^{\prime}\right)+J_{x}\left(\mathbf{r}^{\prime}\right) \frac{1}{k^{2}} \frac{\partial}{\partial x^{\prime}} \frac{\partial}{\partial z^{\prime}}\right.} \\
& \left.+\frac{J_{z}\left(\mathbf{r}^{\prime}\right)}{k^{2}} \frac{\partial^{2}}{\partial z^{\prime 2}}\right] H_{0}^{(1)}\left(k\left|\mathbf{r}-\mathbf{r}^{\prime}\right|\right)\left[1+\left(\frac{\mathrm{d} D}{\mathrm{~d} x^{\prime}}\right)^{2}\right]^{1 / 2} \mathrm{~d} x^{\prime},
\end{aligned}
$$

for which $\mathbf{n}$ is the local outward normal to the surface,

$$
\mathbf{n}=\left(-\frac{\mathrm{d} D}{\mathrm{~d} x}, 0,1\right) /\left[1+\left(\frac{\mathrm{d} D}{\mathrm{~d} x^{\prime}}\right)^{2}\right]^{1 / 2},
$$

and the current density $\mathbf{J}$ has the property that

$$
\mathbf{J} \cdot \mathbf{n}=0
$$

so that

$$
-J_{x} \mathrm{~d} D / \mathrm{d} x^{\prime}+J_{z}=0 .
$$

By introducing Eqs. (A11) into Eq. (A1) and taking Eq. (A14) into account, we readily obtain, for $s$ waves [for which $\mathbf{E}^{(i)}$ is given by Eq. (1)], that $J_{x}=J_{z}=0$. Therefore, for $s$ waves, the extinction theorem yields

$$
\begin{aligned}
E^{(i)} & \exp \left[\left(K_{0} x-q_{0} z\right)\right] \\
\quad= & \frac{\pi k}{c} \int_{-\infty}^{\infty} J_{y}\left(\mathbf{r}^{\prime}\right) H_{0}^{(1)}\left(k\left|\mathbf{r}-\mathbf{r}^{\prime}\right|\right)\left[1+\left(\frac{\mathrm{d} D}{\mathrm{~d} x}\right)^{2}\right]^{1 / 2} \mathrm{~d} x^{\prime} .
\end{aligned}
$$

Similarly, by introducing Eqs. (A11) into Eq. (A2), we obtain the scattered field above the surface $z=D(x)$ :

$$
E_{y}^{(s)}\left(\mathbf{r}_{>}\right)=\frac{\pi k}{c} \int_{-\infty}^{\infty} J_{y}\left(\mathbf{r}^{\prime}\right) H_{0}^{(1)}\left(k\left|\mathbf{r}-\mathbf{r}^{\prime}\right|\right)\left[1+\left(\frac{\mathrm{d} D}{\mathrm{~d} x^{\prime}}\right)^{2}\right]^{1 / 2} \mathrm{~d} x^{\prime} .
$$

For $p$ waves and the magnetic field, Eqs. (A5) and (A7) yield $J_{y}=0$ in a similar way. Finally, by expressing $J_{z}$ in terms of $J_{x}$ through Eq. (14), we obtain

$$
\begin{aligned}
{\left[S_{h}(\mathbf{r})\right]_{y} } & =\frac{4 \pi^{2} i k}{c} \int_{-\infty}^{\infty} J_{x}\left(\mathbf{r}^{\prime}\right) H_{1}^{(1)}\left(k\left|\mathbf{r}-\mathbf{r}^{\prime}\right|\right) \\
& \times \frac{\left(z-z^{\prime}\right)-\frac{\mathrm{d} D}{\mathrm{~d} x^{\prime}}\left(x-x^{\prime}\right)}{\left[\left(x-x^{\prime}\right)^{2}+\left(z-z^{\prime}\right)^{2}\right]^{1 / 2}}\left[1+\left(\frac{\mathrm{d} D}{\mathrm{~d} x^{\prime}}\right)^{2}\right]^{1 / 2} \mathrm{~d} x^{\prime} .
\end{aligned}
$$

In Eq. (A17), $H_{1}{ }^{(1)}$ is the first-order Hankel function of the first kind.

For $p$ waves, therefore, Eqs. (8), (A5), and (A17) give the expression for the extinction theorem integral equation for $J_{x}$ :

$$
\begin{gathered}
H^{(i)} \exp \left[i\left(k_{0} x-q_{0} z\right)\right]=-\frac{\pi i k}{c} \int_{-\infty}^{\infty} J_{x}\left(\mathbf{r}^{\prime}\right) H_{1}^{(1)}\left(k\left|\mathbf{r}-\mathbf{r}^{\prime}\right|\right) \\
\times \frac{\left(z-z^{\prime}\right)-\frac{\mathrm{d} D}{\mathrm{~d} x^{\prime}}\left(x-x^{\prime}\right)}{\left[\left(x-x^{\prime}\right)^{2}+\left(z-z^{\prime}\right)^{2}\right]^{1 / 2}}\left[1+\left(\frac{\mathrm{d} D}{\mathrm{~d} x^{\prime}}\right)^{2}\right]^{1 / 2} \mathrm{~d} x^{\prime} .
\end{gathered}
$$

The singularity of $H_{1}^{(1)}\left(k\left|\mathbf{r}-\mathbf{r}^{\prime}\right|\right)$ at $\mathbf{r}=\mathbf{r}^{\prime}$ is not integrable [in contrast to that of $H_{0}^{(1)}\left(k\left|\mathbf{r}-\mathbf{r}^{\prime}\right|\right)$ for $s$ waves]. In addition, we must consider the geometric factor in Eq. (A18). This makes it necessary to evaluate the principal value of this integral. The procedure was developed in Ref. 48 and leads to the following expression for Eq. (A18):

$$
\begin{aligned}
H^{(i)} & \exp \left[i\left(K_{0} x-q_{0} z\right)\right] \\
& =-\frac{\pi i k}{c}\left\{\frac{2 i}{k} \Delta\left(\mathbf{r}-\mathbf{r}^{\prime}\right)+\left[1-\Delta\left(\mathbf{r}-\mathbf{r}^{\prime}\right)\right]\right. \\
& \times \int_{-\infty}^{+\infty} J_{x}\left(\mathbf{r}^{\prime}\right) H_{1}^{(1)}\left(k \mid \mathbf{r}-\mathbf{r}^{\prime}\right) \\
& \left.\times \frac{\left(z-z^{\prime}\right)-\frac{\mathrm{d} D}{\mathrm{~d} x^{\prime}}\left(x-x^{\prime}\right)}{\left[\left(x-x^{\prime}\right)^{2}+\left(z-z^{\prime}\right)^{2}\right]^{1 / 2}}\left[1+\left(\frac{\mathrm{d} D}{\mathrm{~d} x^{\prime}}\right)^{2}\right]^{1 / 2} \mathrm{~d} x^{\prime}\right\},
\end{aligned}
$$

where

$$
\Delta\left(\mathbf{r}-\mathbf{r}^{\prime}\right)= \begin{cases}1 & \mathbf{r}=\mathbf{r}^{\prime} \\ 0 & \mathbf{r} \neq \mathbf{r}^{\prime}\end{cases}
$$


Similarly, from Eqs. (A17) and (A6) we obtain the scattered magnetic vector:

$$
\begin{aligned}
& H_{y}{ }^{(s)}\left(\mathbf{r}_{>}\right)=\frac{\pi i k}{c} \int_{-\infty}^{+\infty} J_{x}\left(\mathbf{r}^{\prime}\right) H_{1}^{(1)}\left(k\left|\mathbf{r}_{>}-\mathbf{r}^{\prime}\right|\right) \\
& \quad \times \frac{\left(z_{>}-z^{\prime}\right)-\frac{\mathrm{d} D}{\mathrm{~d} x^{\prime}}\left(x_{>}-x^{\prime}\right)}{\left[\left(x_{>}-x^{\prime}\right)^{2}+\left(z_{>}-z^{\prime}\right)^{2}\right]^{1 / 2}}\left[1+\left(\frac{\mathrm{d} D}{\mathrm{~d} x^{\prime}}\right)^{2}\right]^{1 / 2} \mathrm{~d} x^{\prime} .
\end{aligned}
$$

\section{APPENDIX B}

For periodic surfaces we can take advantage of the pseudoperiodicity of the induced electric current density ${ }^{29,49}$ :

$$
\mathbf{J}(x+a)=\mathbf{J}(x) \exp \left(i K_{0} a\right),
$$

for which $a$ is the grating period and $K_{0}$ is given according to Eq. (2a).

The extinction theorem and the diffracted field above the surface are considered first for $s$ waves.

By using the expansion of the Hankel function into plane waves (similar to the Weyl expansion), we obtain, from Eq. (A16),

$$
\begin{aligned}
E_{y}{ }^{(s)}\left(\mathbf{r}_{>}\right)=\frac{-\pi k}{c} \int_{-\infty}^{+\infty} & \mathrm{d} x^{\prime} J_{y}\left(\mathbf{r}^{\prime}\right) \frac{1}{\pi} \int_{-\infty}^{\infty} \mathrm{d} K \exp \left[i K\left(x-x^{\prime}\right)\right] \\
& \times \frac{\exp \left(i q\left|z-z^{\prime}\right|\right)}{q}\left[1+\left(\frac{\mathrm{d} D}{\mathrm{~d} x^{\prime}}\right)^{2}\right]^{1 / 2} .
\end{aligned}
$$

In Eq. (B2) $K$ and $q$ are related by

$$
\begin{aligned}
q & =\left(k^{2}-K^{2}\right)^{1 / 2} & \text { if } K \leq k \text { (homogeneous waves) } \\
& =i\left(K^{2}-k^{2}\right)^{1 / 2} & \text { if } K>k \text { (evanescent waves). }
\end{aligned}
$$

The $x^{\prime}$ integral in Eq. (B2) may be decomposed into an infinite sum of integrals, each extended over one period. Hence, if we use Eq. (B1), then Eq. (B2) yields

$$
\begin{aligned}
E_{y}^{(s)}\left(\mathbf{r}_{>}\right)= & -\frac{k}{c} \int_{0}^{a} J_{y}\left(\mathbf{r}^{\prime}\right)\left\{\int _ { - \infty } ^ { + \infty } \mathrm { d } K \operatorname { e x p } \left[i K\left(x-x^{\prime}\right)\right.\right. \\
& \times \frac{\exp \left[i q\left|z-D\left(x^{\prime}\right)\right|\right]}{q} \\
& \left.\times \sum_{l=-\infty}^{\infty} \exp \left[i\left(K_{0}-K\right) l a\right]\left[1+\left(\frac{\mathrm{d} D}{\mathrm{~d} x^{\prime}}\right)^{2}\right]^{1 / 2}\right\} \mathrm{d} x^{\prime} .
\end{aligned}
$$

We can now use the following equation, ${ }^{51}$ which expresses the Fourier transform of the Dirac comb ${ }^{52}$ :

$$
\sum_{l=-\infty}^{\infty} \exp \left[-i\left(K-K_{0}\right) l a\right]=\frac{2 \pi}{a} \sum_{n=-\infty}^{\infty} \delta\left[K-\left(K_{0}+\frac{2 \pi n}{a}\right)\right] \text {. }
$$

Equation (B4) then gives

$$
\begin{aligned}
E_{y}^{(s)}\left(\mathbf{r}_{>}\right)= & \frac{2 \pi k}{c a} \sum_{n=-\infty}^{\infty} \int_{0}^{a} J_{y}\left(\mathbf{r}^{\prime}\right) \frac{1}{q_{n}} \exp \left[i K_{n}\left(x-x^{\prime}\right)\right] \\
& \times \exp \left[i q_{n}\left|z-D\left(x^{\prime}\right)\right|\right]\left[1+\left(\frac{\mathrm{d} D}{\mathrm{~d} x^{\prime}}\right)^{2}\right]^{1 / 2} \mathrm{~d} x^{\prime} .
\end{aligned}
$$

In Eq. (B6) we have

$$
K_{n}=K_{0}+2 \pi n / a,
$$

which is a well-known expression for diffraction gratings. Also, we have

$$
\begin{array}{rlrl}
q_{n} & =\left(k^{2}-K_{n}\right)^{1 / 2} & & \text { if } K_{n} \leq k \text { (homogeneous waves) } \\
& =i\left(K_{n}-k^{2}\right)^{1 / 2} & \text { if } K_{n}>k \text { (evanescent waves). }
\end{array}
$$

Finally, for points $\mathbf{r}_{>}$such that $z_{>}>D_{\max }$, where $D_{\max }$ is the highest value of $z=D(x)$, we can write $\left|z-D\left(x^{\prime}\right)\right|=z-$ $D\left(x^{\prime}\right)$; then Eq. (B6) takes the form

$$
E_{y}{ }^{(s)}\left(\mathbf{r}_{>}\right)=-\sum_{n=-\infty}^{\infty} A_{n} \exp \left[i\left(K_{n} x+q_{n} z\right)\right],
$$

where the diffraction-order amplitudes are

$$
\begin{aligned}
A_{n}=\frac{2 \pi k}{c a q_{n}} \int_{0}^{a} J_{y}\left[x^{\prime}, D\left(x^{\prime}\right)\right] \exp [ & -i\left(K_{n} x^{\prime}+q_{n} D\left(x^{\prime}\right)\right] \\
\times & {\left[1+\left(\frac{\mathrm{d} D}{\mathrm{~d} x^{\prime}}\right)^{2}\right]^{1 / 2} \mathrm{~d} x^{\prime} }
\end{aligned}
$$

and the diffracted intensity for each order, normalized to the incident intensity $\mid E^{(i) \mid 2}$, is given by

$$
I_{n}^{s}=\frac{q_{n}}{q_{0}} \frac{\left|A_{n}\right|^{2}}{\left|E^{(i)}\right|^{2}}
$$

The calculation of the electric current $J_{y}$ by means of the extinction theorem can be done by using the following expression, which can be obtained by a method analogous to that used for Eq. (B6):

$$
\begin{aligned}
E^{(i)} \exp \left[i \left(K_{0} x\right.\right. & \left.\left.-q_{0} z\right)\right]=\frac{\pi k}{c} \int_{0}^{a} J_{y}\left[x^{\prime}, D\left(x^{\prime}\right)\right] \\
& \times\left[\sum _ { l = - \infty } ^ { \infty } H _ { 0 } ^ { ( 1 ) } \left(k \left\{\left[x-\left(x^{\prime}+l a\right)\right]^{2}\right.\right.\right. \\
& \left.\left.\left.+\left[z-D\left(x^{\prime}\right)\right]^{2}\right\}^{1 / 2}\right) \exp \left(i K_{0} l a\right)\right] \\
& \times\left[1+\left(\frac{\mathrm{d} D}{\mathrm{~d} x^{\prime}}\right)^{2}\right]^{1 / 2} \mathrm{~d} x^{\prime} .
\end{aligned}
$$

For $p$ waves we obtain in a similar fashion the expression for the extinction theorem that yields the boundary condition (from which we can obtain the electric current density),

$$
\begin{aligned}
& H^{(i)} \exp \left[i\left(K_{0} x-q_{0} z\right)\right]=-\frac{\pi i k}{c} \int_{0}^{a} J_{x}\left[x^{\prime}, D\left(x^{\prime}\right)\right] \\
& \times\left[\sum_{l=-\infty}^{\infty} \frac{\left[z-D\left(x^{\prime}\right)\right]-\frac{\mathrm{d} D}{\mathrm{~d} x^{\prime}}\left[x-\left(x^{\prime}+l a\right)\right]}{\left\{\left(x-\left(x^{\prime}+l a\right)\right]^{2}+\left[z-D\left(x^{\prime}\right)\right]^{2}\right\}^{1 / 2}}\right. \\
& \left.\times \exp \left(i K_{0} l a\right) H_{1}^{(1)}\left(k\left\{\left[x-\left(x^{\prime}+l a\right)\right]^{2}+\left(z-D\left(x^{\prime}\right)\right]^{2}\right\}^{1 / 2}\right)\right] \\
& \times\left[1+\left(\frac{\mathrm{d} D}{\mathrm{~d} x^{\prime}}\right)^{2}\right]^{1 / 2} \mathrm{~d} x^{\prime}, \quad(\mathrm{B} 12)
\end{aligned}
$$

and the diffracted magnetic vector for points $\mathbf{r}_{>}$such that $z_{>}$ $>D_{\max }$ 


$$
H_{y}{ }^{(s)}\left(\mathbf{r}_{>}\right)=\sum_{n=-\infty}^{\infty} B_{n} \exp \left[i\left(K_{n} x+q_{n} z\right)\right],
$$

where the amplitude of each diffraction order is

$$
\begin{aligned}
B_{n}=-\frac{2 \pi k}{c a q_{n}} \int_{0}^{a} & J_{x}\left[x^{\prime}, D\left(x^{\prime}\right)\right] \exp \left\{-i\left[K_{n} x^{\prime}+q_{n} D\left(x^{\prime}\right)\right]\right\} \\
& \times\left(\frac{\mathrm{d} D}{\mathrm{~d} x^{\prime}} K_{n}-q_{n}\right)\left[1+\left(\frac{\mathrm{d} D}{\mathrm{~d} x^{\prime}}\right)^{2}\right]^{1 / 2} \mathrm{~d} x^{\prime} .
\end{aligned}
$$

Thus the diffracted intensity for each order, normalized to the incident intensity, $\left|H^{(i)}\right| 2$, for $p$ waves is given by

$$
I_{n}{ }^{p}=\frac{q_{n}}{q_{0}} \frac{\left|B_{n}\right|^{2}}{\left|E^{(i)}\right|^{2}} \cdot
$$

Both $I_{n}{ }^{p}$ and $I_{n}{ }^{s}$ must satisfy the unitarity condition,

$$
\sum_{n} I_{n}^{\{p ;}=1
$$

where the sum is made over only the propagating orders.

\section{ACKNOWLEDGMENTS}

This research was supported by the Comision Interministerial de Ciencia y Tecnología under grant PB0278. The authors would also like to acknowledge the UK-Spain Integrated Action program. Discussions with J. C. Dainty, A. T. Friberg, and M. J. Kim were stimulating. J. M. Soto-Crespo acknowledges a grant from Ministerio de Educación y Ciencia.

\section{REFERENCES}

1. P. Beckmann and A. Spizzichino, The Scattering of Electromagnetic Waves from Rough Surfaces (Macmillan, New York, 1963).

2. P. Beckmann, "Scattering of light by rough surfaces," in Progress in Optics VI, E. Wolf, ed. (North Holland, Amsterdam, 1961), pp. 55-69.

3. F. G. Bass and I. M. Fuks, Wave Scattering from Statistically Rough Surfaces (Pergamon, Oxford, 1979)

4. J. C. Leader, "Bidirectional scattering of electromagnetic waves from rough surfaces," J. Appl. Phys. 42, 4808-4816 (1971).

5. S. O. Rice, "Reflection of electromagnetic waves from slightly rough surfaces," Commun. Pure Appl. Math. 4, 351-378 (1951).

6. J.C. Leader, "The relationship between the Kirchhoff approach and small perturbation analysis in rough surface scattering theory," IEEE Trans. Antennas Propag. AP-19, 786-788 (1971)

7. G. R. Valenzuela, J. W. Wright, and J. C. Leader, "Comments on the relationship between the Kirchhoff approach and small perturbation analysis in rough surface scattering theory," IEEE Trans. Antennas Propag. AP-20, 536-539 (1972).

8. G. R. Valenzuela, "Depolarization of EM waves by slightly rough surfaces," IEEE Trans. Antennas Propag. AP-15, 522557 (1967)

9. D. N. Pattanayak and E. Wolf, "General form and a new interpretation of the Ewald-Oseen extinction theorem," Opt. Commun. 6, 217-220 (1972).

10. F. Toigo, A. Marvin, V. Celli, and N. R. Hill, "Optical properties of rough surfaces: general theory and the small roughness limit," Phys. Rev. 15, 5618-5626 (1977).

11. G. S. Agarwal, "Interaction of electromagnetic waves at rough dielectric surfaces," Phys. Rev. B 15, 2371-2383 (1977).

12. M. Nieto-Vesperinas and N. Garcia, "A detailed study of the scattering of scalar waves from random rough surfaces," Opt. Acta 28, 1651-1672 (1981).
13. M. Nieto-Vesperinas, "Depolarization of EM waves scattered from slightly rough random surfaces: a study by means of the extinction theorem," J. Opt. Soc. Am. 72, 539-547 (1982).

14. J. Shen and A. Maradudin, "Multiple scattering of waves from random rough surfaces," Phys. Rev. B 22, 4234-4240 (1980).

15. D. P. Winebrenner and A. Ishimaru, "Application of the phaseperturbation technique to randomly rough surfaces," J. Opt. Soc. Am. A 2, 2285-2294 (1985).

16. D. Maystre and J. P. Rossi, "Implementation of a rigorous vector theory of speckle for two-dimensional microrough surfaces," J. Opt. Soc. Am. A 3, 1276-1282 (1986).

17. G. S. Brown, "A comparison of approximate theories for scattering from rough surfaces," Wave Motion 7, 195-205 (1985).

18. J. A. de Santo and G. S. Brown, "Analytical techniques for multiple scattering from rough surfaces," in Progress in Optics XXIII, E. Wolf, ed. (North-Holland, Amsterdam, 1986), pp. 362.

19. E. R. Mendez and K. A. O'Donnell, "Observation of depolarization and backscattering enhancement in light scattering from Gaussian random surfaces," Opt. Commun. 61, 91-95 (1987).

20. K. A. O'Donnell and E. R. Mendez, "Experimental study of scattering from characterized random surfaces," J. Opt. Soc. Am. A 4, 1194-1205 (1987).

21. Y. Kuga and A. Ishimaru, "Retroreflectance from a dense distribution of spherical particles," J. Opt. Soc. Am. A 1, 831-835 (1984).

22. L. Tsang and A. Ishimaru, "Backscattering enhancement of random discrete scatterers," J. Opt. Soc. Am. A 1, 836-839 (1984).

23. H. P. Van Albada and A. Lagendijk, "Observation of weak localization of light in a random medium," Phys. Rev. Lett. 55, 2692-2695 (1983).

24. P. E. Wolf and G. Maret, "Weak localization and coherent backscattering of photons in disordered media," Phys. Rev. Lett. 55, 2696-2699 (1985).

25. M. Kaveh, M. Rosenbluth, M. Edrei, and I. Freund, "Weak localization and light scattering from disordered solids," Phys. Rev. Lett. 57, 2049-2052 (1986).

26. V. Celli, A. A. Maradudin, A. M. Marvin, and A. R. McGurn, "Some aspects of light scattering from a randomly rough metal surface," J. Opt. Soc. Am. A 2, 2225-2239 (1985); see also P. Tran and V. Celli, "Monte Carlo calculation of backscattering enhancement for a randomly rough grating," J. Opt. Soc. Am. A 5, 1635-1637 (1988)

27. M. Nieto-Vesperinas and J. M. Soto-Crespo, "Monte Carlo simulations for scattering of electromagnetic waves from perfectly conductive random rough surfaces," Opt. Lett. 979-981 (1987).

28. E. Bahar and M. A. Fitzwater, "Full wave theory and controlled optical experiments for enhanced scatter and depolarization by random rough surfaces," Opt. Commun. 63, 355-360 (1987).

29. R. Petit, ed., "Electromagnetic theory of gratings," Vol. 22 of Topics in Current Physics (Springer-Verlag, Berlin, 1980).

30. N. Garcia and N. Cabrera, "New method for solving the scattering of waves from a periodic hard surface," Phys. Rev. B 18, 576589 (1978).

31. See Ref. 29, pp. 159-225.

32. D. Y. Tseng, A. Hessel, and A. A. Oliner, "Scattering by a multimode corrugated structure with application to $p$ type Wood anomalies," Alta Freq. 38 (special issue: URSI Symposium on EM Waves), 82-88 (1969).

33. A. Hessel and A. A. Oliner, "A new theory of Wood's anomalies on optical gratings," Appl. Opt. 4, 1275-1279 (1965).

34. A. Hessel, J. Schmoys, and D. Y. Tseng, "Bragg-angle blazing of diffraction gratings," J. Opt. Soc. Am. 65, 380-384 (1975).

35. N. Garcia and A. A. Maradudin, "Exact calculations of the diffraction of s-polarized electromagnetic radiation from largeamplitude dielectric gratings," Opt. Commun. 45, 301-306 (1983).

36. A. Hessel and A. A. Oliner, "Wood's anomaly effects on gratings of large amplitude," Opt. Commun. 59, 327-330 (1986).

37. M. Nieto-Vesperinas and J. M. Soto-Crespo, "Light diffracted intensities from deep gratings," Phys. Rev. B 38, 7250-7259 (1988).

38. M. Abramowitz and I. A. Stegun, Handbook of Mathematical Functions (Dover, New York, 1965), p. 364. 
39. R. M. Axline and A. K. Fung, "Numerical computation of scattering from a perfectly conducting random surface," IEEE Trans. Antennas Propag. AP-16, 482-488 (1978); H. L. Chan and A. K. Fung, "A numerical study of the Kirchhoff approximation in horizontally polarized backscattering from a random surface," Radio Sci. 13, 811-818 (1978); A. K. Fung and M. F. Chen, "Numerical simulation of scattering from simple and composite random surfaces," J. Opt. Soc. Am. A 2, 2274-2284 (1985).

40. N. Garcia, "Exact calculations of $p$-polarized electromagnetic fields incident on grating surfaces: Surface polaritons resonances," Opt. Commun. 45, 307-310 (1983), and references therein.

41. J. C. Dainty and M. J. Kim, Imperial College, London, UK (personal communication, 1988).

42. J. Renau and J. A. Collinson, "Measurements of electromagnetic backscattering from known, rough surfaces," Bell Syst. Tech. J. 44, 2203-2226 (1965).

43. M. Nieto-Vesperinas, "Radiometry of rough surfaces," Opt. Acta 29, 961-971 (1982).

44. J. M. Soto-Crespo and M. Nieto-Vesperinas, "Enhancement of all antispecular orders from deep gratings," Opt. Commun. (to be published).
45. D. Maystre, "Electromagnetic scattering from perfectly conducting rough surfaces in the resonance region," IEEE Trans. Antennas. Propag. AP-31, 885-895 (1983).

46. V. Garibaldi, A. C. Levi, R. Spadacini, and G. E. Tommei, "Quantum theory of atom-surface scattering: diffraction and rainbow," Surf. Sci. 48, 649-675 (1975).

47. E. I. Thorsos, "The validity of the Kirchhoff approximation for rough surface scattering using a Gaussian roughness spectrum," J. Acoust. Soc. Am. 83, 78-92 (1988).

48. M. G. Andreasen, "Scattering from parallel metallic cylinders with arbitrary cross sections," IEEE Trans. Antennas Propag. AP-12, 746-754 (1964).

49. D. Maystre, "Rigorous vector theories of diffraction gratings," in Progress in Optics XXI, E. Wolf, ed. (North-Holland, Amsterdam, 1984).

50. See Ref. 38, p. 823.

51. N. R. Hill and V. Celli, "Multiple hits in atom-surface diffraction," Surf. Sci. 75, 577-591 (1978).

52. R. N. Bracewell, The Fourier Transform and Its Applications (McGraw-Hill, New York, 1978), p. 214. 